\title{
A Distinguisher for High Rate McEliece Cryptosystems
}

\author{
Jean-Charles Faugère, Valérie Gauthier-Umaña, Ayoub Otmani, Ludovic Perret, Jean-Pierre Tillich
}

\begin{abstract}
The Goppa Code Distinguishing (GD) problem consists in distinguishing the matrix of a Goppa code from a random matrix. The hardness of this problem is an assumption to prove the security of code-based cryptographic primitives such as McEliece's cryptosystem. Up to now, it is widely believed that the GD problem is a hard decision problem. We present the first method allowing to distinguish alternant and Goppa codes over any field. Our technique can solve the GD problem in polynomial-time provided that the codes have sufficiently large rates. The key ingredient is an algebraic characterization of the key-recovery problem. The idea is to consider the rank of a linear system which is obtained by linearizing a particular polynomial system describing a key-recovery attack. It appears that this dimension depends on the type of code considered. Explicit formulas derived from extensive experimentations for the rank are provided for "generic" random, alternant, and Goppa codes over any field. Finally, we give theoretical explanations of these formulas in the case of random codes, alternant codes over any field of characteristic two and binary Goppa codes.
\end{abstract}

\section{Index Terms}

McEliece cryptosystem, CFS signature, Algebraic cryptanalysis, Goppa Code Distinguishing problem.

\section{INTRODUCTION}

$\mathbf{T}$ HIS paper ${ }^{1}$ investigates the difficulty of the Goppa Code Distinguishing (GD) problem which first appeared in [2]. This is a decision problem that aims at recognizing a generator matrix of a binary Goppa code from a randomly drawn binary matrix. Up to now, it is assumed that no polynomial time algorithm exists that distinguishes a generator matrix of a Goppa code from a randomly picked generator matrix.

The main motivation for introducing the GD problem is to formally relate the problem of decoding a random linear code to the security of the McEliece public-key cryptosytem [3]. Since its apparition, this cryptosystem has withstood many attacks and after more than thirty years now, it still belongs to the very few unbroken public key cryptosystems. This situation substantiates the claim that inverting the encryption function, and in particular recovering the private key from public data, is intractable. The classical methods for inverting the McEliece encryption function without finding a trapdoor all resort to the use of the best general decoding algorithms [4]-[11]. All these algorithms, whose time complexity is exponential (in the length), attempt to solve the long-standing problem of decoding random linear code [12]. They also assume (implicitly or explicitly) that there does not exist an algorithm that is able to decode more efficiently McEliece public keys. Note that if ever such an algorithm exists, it would permit to solve the GD problem.

On the other hand, no significant breakthrough has been observed with respect to the problem of recovering the private key [13], [14]. This has led to state that the generator matrix of a binary Goppa code does not disclose any visible structure that an attacker could exploit. This is strengthened by the fact that Goppa codes share many characteristics with random codes. For instance they asymptotically meet the Gilbert-Varshamov bound. They also have a trivial permutation group, etc. Hence, the hardness of the GD problem has become a classical belief, and as a consequence, a de facto assumption to prove the semantic security in the standard model (IND-CPA in [15] and IND-CCA2 in [16]), and the security in the random oracle model against existential forgery [2], [17] of the signature scheme [2].

We present a deterministic polynomial-time distinguisher for codes whose rate is close to 1 . This includes in particular codes encountered with the signature scheme CFS ( [2], [18]). We emphasize that our method can distinguish codes also used in McEliece's encryption scheme. For instance, the binary Goppa code obtained with $m=13$ and $r=19$ corresponding to a 90-bit security key is distinguishable. We provide an asymptotic formula for the smallest rate $R_{\text {crit }}$ for which one can

J.-C. Faugère and L. Perret are with

INRIA, Paris-Rocquencourt Center, POLSYS Project,

UPMC Univ Paris 06, UMR 7606, LIP6, F-75005, Paris, France,

CNRS, UMR 7606, LIP6, F-75005, Paris, France,

e-mail: jean-charles.faugere@ inria.fr, ludovic.perret@lip6.fr

Valérie Gauthier-Umaña is with Departamento de Matemáticas, Universidad de los Andes, Bogotá, Colombia, email: ve.gauthier@uniandes.edu.co

A. Otmani is with LITIS - Université de Rouen, Avenue de l'Université, F-76801 Saint-Étienne-du-Rouvray Cedex, e-mail: ayoub.otmani@univ-rouen.fr

J.-P. Tillich is with SECRET Project - INRIA Rocquencourt, Domaine de Voluceau, B.P. 105, 78153 Le Chesnay Cedex - France, e-mail: jeanpierre.tillich@inria.fr

${ }^{1}$ A preliminary version [1] of this paper has been presented in IEEE Information Theory Workshop (ITW 2011), Paraty, Brazil, Oct. 16-20 2011. 
distinguish a $q$-random code from a $q$-ary alternant or Goppa code (Theorem 3). If $q$ is fixed and assuming that the length is $q^{m}$ then when $m$ tends to infinity, we have:

$$
R_{\text {crit }}=1-\sqrt{\frac{2 m \log q}{q^{m} \log m}}(1+o(1)) .
$$

where all logarithms are taken to base 2 .

Our distinguisher is based on the algebraic attack developed against compact variants of McEliece [19]. In this approach, the key-recovery problem is transformed into the one of solving an algebraic system. By using a linearization technique, we are able to derive a linear system whose rank is different from what one would expect in the random case. More precisely, we observe experimentally that this defect in the rank is directly related to the type of codes. We provide explicit formulas for "generic" random, alternant, and Goppa codes over any alphabet. We performed extensive experiments to confirm that the formulas are accurate. Eventually, we prove the formula in the random case and give explanations in the case of alternant codes over any field of characteristic two and binary Goppa codes.

However, the existence of our distinguisher does not undermine the security of primitives based on Goppa codes, but basically, it proves that the GD assumption is false for some parameters, and consequently should be used with great care as an assumption for a security reduction.

The paper is organized as follows. After recalling basic notions in coding-theory in Section II, we introduce in Section III our algebraic distinguisher which is basically the dimension of the solution space of a linear system that is deduced by linearization from the algebraic system that any McEliece cryptosystem must satisfy. We then provide explicit formulas that predicts the behavior of the distinguisher coming from experiments. In Section IV and Section V, we give explanations of the formulas for alternant and binary Goppa codes. In Section VI, we give a proof of its typical behavior in the random case. Lastly, we conclude over the cryptographic implications the distinguisher induces and we deduce an asymptotic formula for the smallest rate for which we can distinguish a random code from an alternant code or a Goppa code.

\section{Code-Based Public-Key CRyptography}

The problem of decoding random linear codes is a potential candidate for building public-key cryptographic primitives such as an encryption scheme. McEliece [3] was the first to use this problem in public-key cryptography. The idea is to start from afamily of codes equipped with a polynomial-time decoding algorithm. The fundamental concept of is to consider two equivalent representations of a code: one should facilitate the decoding, whereas the decoding should be infeasible from the other one. Although his design principle is general, he explicitly advocated to use binary Goppa codes [20].

\section{A. Coding Theory Background}

Code-based public-key cryptography focuses on linear codes that have a polynomial time decoding algorithm. We recall that a $q$-ary (linear) code $\mathscr{C}$ over the finite field $\mathbb{F}_{q}$ of $q$ elements defined by a $k \times n$ matrix $\boldsymbol{G}$ (with $k \leqslant n$ ) whose entries belong to $\mathbb{F}_{q}$ is the vector space spanned by its rows i.e.,

$$
\mathscr{C} \stackrel{\text { def }}{=}\left\{\boldsymbol{u} \boldsymbol{G} \mid \boldsymbol{u} \in \mathbb{F}_{q}^{k}\right\}
$$

The length of $\mathscr{C}$ is $n$ and its rate is the ratio $R \stackrel{\text { def }}{=} k / n$. The role of decoding algorithms is to correct errors of prescribed weight. We say that a decoding algorithm corrects $r$ errors if it recovers $\boldsymbol{u}$ from the knowledge of $\boldsymbol{u} \boldsymbol{G}+\boldsymbol{e}$ for all possible $e \in \mathbb{F}_{q}^{n}$ of weight at most $r$.

One famous family of codes is the one of binary Goppa codes. It belongs to the more general class of alternant codes ( [21, Chap. 12, p. 365]). The main well-known feature of an alternant code is the possibility of being decoded in polynomial time. It is more convenient to describe this class through a parity-check matrix over an extension field $\mathbb{F}_{q^{m}}$ of $\mathbb{F}_{q}$ over which the code is defined. We recall that a parity-check matrix $\boldsymbol{H}$ of a $q$-ary code $\mathscr{C}$ is defined as a matrix such that:

$$
\mathscr{C} \stackrel{\text { def }}{=}\left\{c \in \mathbb{F}_{q}^{n} \mid \boldsymbol{H} \boldsymbol{c}^{T}=\mathbf{0}\right\} \text {. }
$$

where the symbol ${ }^{T}$ means the transpose operation. For $q$-ary alternant codes of length $n \leqslant q^{m}$, there exists a parity-check matrix with a very special form related to rectangular Vandermonde matrices:

$$
\boldsymbol{V}_{r}(\boldsymbol{x}, \boldsymbol{y}) \stackrel{\text { def }}{=}\left(\begin{array}{lll}
y_{1} & \cdots & y_{n} \\
y_{1} x_{1} & \cdots & y_{n} x_{n} \\
\vdots & & \vdots \\
y_{1} x_{1}^{r-1} & \cdots & y_{n} x_{n}^{r-1}
\end{array}\right)
$$

where $\boldsymbol{x}=\left(x_{1}, \ldots, x_{n}\right)$ and $\boldsymbol{y}=\left(y_{1}, \ldots, y_{n}\right)$ are in $\mathbb{F}_{q^{m}}^{n}$. 
Definition 1 (Alternant code): A $q$-ary alternant code of order $r$ associated to $\boldsymbol{x}=\left(x_{1}, \ldots, x_{n}\right) \in \mathbb{F}_{q^{m}}^{n}$ where all $x_{i}$ 's are distinct and $\boldsymbol{y}=\left(y_{1}, \ldots, y_{n}\right) \in\left(\mathbb{F}_{q^{m}}^{*}\right)^{n}$ denoted by $\mathscr{A}_{r}(\boldsymbol{x}, \boldsymbol{y})$ is

$$
\left\{\boldsymbol{c} \in \mathbb{F}_{q}^{n} \mid \boldsymbol{V}_{r}(\boldsymbol{x}, \boldsymbol{y}) \boldsymbol{c}^{T}=\mathbf{0}\right\} .
$$

It is well-known that the dimension $k$ of an alternant codes of degree $r$ satisfies $k \geqslant n-r m$. Moreover, a key feature about them is the following property.

Proposition 1: An alternant codes of degree $r$ can decode in polynomial time all errors of weight at most $\frac{r}{2}$ whenever there exists a parity-check matrix in the form $\boldsymbol{V}_{r}\left(\boldsymbol{x}^{*}, \boldsymbol{y}^{*}\right)$ for some vectors $\boldsymbol{x}^{*}$ and $\boldsymbol{y}^{*}$.

Definition 2 (Goppa codes): A $q$-ary Goppa code $\mathscr{G}(\boldsymbol{x}, \gamma)$ associated to a polynomial $\gamma(z) \stackrel{\text { def }}{=} \sum_{i=0}^{r} \gamma_{i} x^{i}$ of degree $r$ over $\mathbb{F}_{q^{m}}$ and an $n$-tuple $\boldsymbol{x}=\left(x_{1}, \ldots, x_{n}\right)$ of distinct elements of $\mathbb{F}_{q^{m}}$ satisfying $\gamma\left(x_{i}\right) \neq 0$ for all $i, 1 \leqslant i \leqslant n$, is the $q$-ary alternant code $\mathscr{A}_{r}(\boldsymbol{x}, \boldsymbol{y})$ of order $r$ with $y_{i}=\gamma\left(x_{i}\right)^{-1}$.

Goppa codes, viewed as alternant codes, inherit a decoding algorithm that corrects up to $\frac{r}{2}$ errors. But in the case of binary Goppa codes, it is possible to correct twice as many errors. The starting point is the following result given in [21, p. 341].

Theorem 1: A binary Goppa code $\mathscr{G}(\boldsymbol{x}, \gamma)$ associated to a Goppa polynomial $\gamma(z)$ of degree $r$ without multiple roots is equal to the alternant code $\mathscr{A}_{2 r}(\boldsymbol{x}, \boldsymbol{y})$ with $y_{i}=\gamma\left(x_{i}\right)^{-2}$.

Corollary 1 ( [22]): There exists a polynomial time algorithm decoding all errors of weight at most $r$ for any Goppa code $\mathscr{G}(\boldsymbol{x}, \gamma)$ where $\gamma(z)$ is of degree $r$ and has no multiple roots.

It is worthwhile recalling that the only requirement for decoding a binary Goppa $\mathscr{G}$ is either to know $\boldsymbol{x}$ and $\gamma(z)$ or to know two vectors $\boldsymbol{x}^{*}$ and $\boldsymbol{y}^{*}$ such that:

$$
\mathscr{G}=\mathscr{A}_{2 r}\left(\boldsymbol{x}^{*}, \boldsymbol{y}^{*}\right) \text {. }
$$

\section{B. Cryptographic Primitives Based on Binary Goppa Codes}

The two most important public schemes that use binary Goppa codes are McEliece's encryption function and CourtoisFiniasz-Sendrier (CFS) [2] signature algorithm. We briefly recall here the general principle of McEliece's scheme. The key generation algorithm picks at random one $k \times n$ generator matrix $\boldsymbol{G}$ of a randomly picked binary Goppa code of $\mathscr{G}$ of degree $r$. The secret key is the decoding algorithm $\mathcal{D}$ associated to $\mathscr{G}$ and the public key is $G$. To encrypt $\boldsymbol{u} \in \mathbb{F}_{2}^{k}$, the sender has to choose a random vector $\boldsymbol{e}$ in $\mathbb{F}_{2}^{n}$ of weight $r$ and computes the ciphertext $\boldsymbol{c} \stackrel{\text { def }}{=} \boldsymbol{u} \boldsymbol{G}+\boldsymbol{e}$. The receiver then recovers the plaintext by applying $\mathcal{D}$ on $c$.

The CFS scheme also relies on binary Goppa codes. A user whose public key is $G$ and who wishes to sign a message $\boldsymbol{x} \in \mathbb{F}_{2}^{k}$ has to compute a string $\boldsymbol{u}$ such that the Hamming weight of $\boldsymbol{x}-\boldsymbol{u} \boldsymbol{G}$ is at most $r$. Anyone (a verifier) can publicly check the validity of a signature. Unfortunately, this approach can only provide signatures for messages $\boldsymbol{x}$ that are within distance $r$ from a codeword $\boldsymbol{u} G$. The CFS scheme prompts to modify the message by appending a counter incremented until the decoding algorithm can find such a signature. The efficiency of this scheme heavily depends on the number of trials. With a binary Goppa codes of length $n=2^{m}$ and and dimension $k=n-m r$, the number of trials is of order $r$ !. So one has to choose a very small $r$ and therefore take a very large $n$ in order to be secure. The code rate is then equal to $1-\frac{1}{2^{m}} m r$ which is quite close to 1 for large $n$ (that is for large values of $m$ ) and moderate values of $r$. For instance, a 80-bit security CFS scheme requires to take $n=2^{21}$ and $r=10$ whereas the McEliece cryptosystem for the same security needs to choose $n=2^{11}$ and $r=32$ ( [18]). Thus one major difference between the McEliece cryptosystem and the CFS scheme lies in the choice of the parameters of the codes.

\section{Goppa Code Distinguishing Problem}

The minimum requirement for an encryption function is that it should be infeasible from a given ciphertext $c$ and public data $^{2}$ like the public key, ciphertexts, etc. to recover the corresponding plaintext $\boldsymbol{x}$. This issue is directly linked to the following computational problem.

Definition 3 (McEliece Problem): Let $\boldsymbol{G}$ be a generator matrix of a binary Goppa code of length $n \leqslant 2^{m}$ and dimension $k=n-r m$ where $m$ and $t$ are positive integers. Let $\boldsymbol{x}$ be a vector from $\mathbb{F}_{2^{m}}^{k}$ and let $\boldsymbol{e}$ be a vector from $\mathbb{F}_{2^{m}}^{n}$ of weight $t$. Finally, we set $\boldsymbol{c} \stackrel{\text { def }}{=} \boldsymbol{x} \boldsymbol{G}+\boldsymbol{e}$. Then the McEliece Problem asks to find $\boldsymbol{x}$ and $\boldsymbol{e}$ only from $\boldsymbol{G}$ and $\boldsymbol{c}$.

One obvious way of solving this problem consists in devising a method that recovers the private key. But, it is also possible to recover a plaintext from a specific ciphertext without resorting to a key-recovery attack. In particular, an attacker against the McEliece scheme would find the plaintext by applying general decoding methods like [3]-[11], [23]-[27] on the public matrix $G$. Such attacks are called decoding attacks. It is possible $\mathcal{G}_{n, k}$ to precise formally all these notions. For any integers $n$ and $k$ with $k \leqslant n$, we denote by $\mathcal{G}_{n, k}$ the set of $k \times n$ generator matrices of binary Goppa codes. Similarly, $\mathcal{R}_{n, k}$ is the set of binary random generator $k \times n$ matrices. The set of words of weight $t$ is denoted by $\mathcal{S}(\mathbf{0}, t)$.

\footnotetext{
${ }^{2}$ This kind of attack is called a Chosen Plaintext Attack (CPA).
} 
Definition 4: An $(T, \varepsilon)$-adversary $\mathbb{A}$ against the McEliece cryptosystem is an algorithm that runs in time at most $T$ such that:

$$
\operatorname{Suc}_{\operatorname{McE}}(\mathbb{A}) \stackrel{\text { def }}{=} \operatorname{Pr}_{\boldsymbol{G}, \boldsymbol{m}, \boldsymbol{e}}\{\mathbb{A}(\boldsymbol{G}, \boldsymbol{m} \boldsymbol{G}+\boldsymbol{e})=(\boldsymbol{m}, \boldsymbol{e})\} \leqslant \varepsilon
$$

where the random choices are made such that $\boldsymbol{G} \in \mathcal{G}_{n, k}, \boldsymbol{m} \in \mathbb{F}_{2}^{k}$ and $\boldsymbol{e} \in \mathcal{S}(\mathbf{0}, t)$.

An algorithm $\mathbb{A}$ is a $(T, \varepsilon)$-decoder if it runs in time at most $T$ such that:

$$
\operatorname{SuC}_{\text {Rand }}(\mathbb{A}) \stackrel{\text { def }}{=} \operatorname{Pr}_{\boldsymbol{G}, \boldsymbol{m}, \boldsymbol{e}}\{\mathbb{A}(\boldsymbol{G}, \boldsymbol{m} \boldsymbol{G}+\boldsymbol{e})=(\boldsymbol{m}, \boldsymbol{e})\} \leqslant \varepsilon
$$

where $\boldsymbol{G} \in \mathcal{R}_{n, k}, \boldsymbol{m} \in \mathbb{F}_{2}^{k}$ and $\boldsymbol{e} \in \mathcal{S}(\mathbf{0}, t)$.

Currently, the only known methods that aim to solve the McEliece problem are based either on an exhaustive search of the private key or on applying very general decoding methods. Both approaches run in exponential time on the length when the rate is fixed. But this situation is a still unsatisfactory because there is no certitude that there does not exist a better way to solve it.

A classical stance is to claim that binary Goppa codes look like random linear codes. It amounts to say that there does not exist a polynomial-time computable quantity which behaves differently depending on whether the code is a Goppa or a random code. Currently, it is an open problem to establish a formal proof that would substantiate the claim that a binary Goppa code is indistinguishable from a random code. This assumption is attractive because it enables to rely on the hardness of decoding a random linear code to prove the security of the McEliece function. This reasoning does make sense because binary Goppa codes share several common aspects ${ }^{3}$ with a randomly picked linear code. Furthermore, all the general decoding algorithms do not exploit the information, even partially, that a matrix describes a "hidden" Goppa code. Based on this, the authors of [2] defined the Goppa Code Distinguishing (GD) problem and stated that "classification issues are in the core of coding theory since its emergence in the 50's. So far nothing significant is known about Goppa codes, more precisely there is no known property invariant by permutation and computable in polynomial time which characterizes Goppa codes. Finding such a property or proving that none exists would be an important breakthrough in coding theory and would also probably seal the fate, for good or ill, of Goppa code-based cryptosystems". We state now precisely the GD problem.

Definition 5 (Goppa Code Distinguishing (GD) Problem): An algorithm $\mathcal{D}: \mathbb{F}_{2}^{k \times n} \rightarrow\{0,1\}$ that takes as input a $k \times n$ matrix $G$ and returns a bit solves the GD problem if it wins the following game:

1) $b \leftarrow\{0,1\}$

2) If $b=1$ then $\boldsymbol{G} \leftarrow \mathcal{G}_{n, k}$ else $G \leftarrow \mathcal{R}_{n, k}$

3) If $\mathcal{D}(\boldsymbol{G})=b$ then $\mathcal{D}$ wins else $\mathcal{D}$ loses.

An algorithm $\mathcal{D}: \mathbb{F}_{2}^{k \times n} \rightarrow\{0,1\}$ is a $(T, \varepsilon)$-distinguisher if it runs in time at most $T$ and such that:

$$
\operatorname{Adv}(\mathcal{D}) \stackrel{\text { def }}{=}\left|\operatorname{Pr}_{\boldsymbol{G} \in \mathcal{G}_{n, k}}\{\mathcal{D}(\boldsymbol{G})=1\}-\operatorname{Pr}_{\boldsymbol{G}}\{\mathcal{D}(\boldsymbol{G})=1\}\right|
$$

There is simple way to construct a distinguisher from an attacker $(T, \varepsilon)$-attacker $\mathbb{A}$ against the McEliece cryptosystem. We denote it by $\mathcal{D}_{\mathbb{A}}$ and it works as follows. On a given input $\boldsymbol{G}$, it randomly picks a couple $(\boldsymbol{m}, \boldsymbol{e})$ among $\mathbb{F}_{2}^{k} \times \mathcal{S}(\mathbf{0}, t)$ and then outputs $\mathcal{D}_{\mathbb{A}}(\boldsymbol{G})=1$ if $\mathbb{A}(\boldsymbol{G}, \boldsymbol{m} \boldsymbol{G}+\boldsymbol{e})=(\boldsymbol{m}, \boldsymbol{e})$ and $\mathcal{D}_{\mathbb{A}}(\boldsymbol{G})=0$ otherwise. The running time of $\mathcal{D}_{\mathbb{A}}(\boldsymbol{G})$ is therefore uppe-bounded by the running time of $\mathbb{A}$ and its advantage is then equal to [28]:

$$
\operatorname{Adv}\left(\mathcal{D}_{\mathbb{A}}\right)=\left|\operatorname{Suc}_{\operatorname{Rand}}(\mathbb{A})-\operatorname{Suc}_{\operatorname{McE}}(\mathbb{A})\right|
$$

This shows that if $\operatorname{Adv}\left(\mathcal{D}_{\mathbb{A}}\right.$ is very small (or negligible) then the chances that an attacker recovers a plaintext are also very small provided that the problem of decoding a random linear code is hard. So the difficulty of the GD problem guarantees that there is no polynomial-time algorithm that solves the McEliece problem.

Until our recent work in [1] and this paper, the only known algorithm for solving GD enumerates binary Goppa codes and tests the code equivalence thanks to the Support Splitting algorithm [29]. This approach runs in time $O\left(\frac{1}{m r} n^{r-1}\right)$ for binary Goppa codes of degree $r$ and length $n$ with $m \leqslant \log _{2} n$. Another possible approach was proposed in [30] which shows that Quantum Fourier Sampling (QFS) can also be used for solving the GD problem. However, it turns out that QFS has a negligible advantage against the GD problem. Thus GD problem seems immune against QFS unlike classical cryptographic problems such as factoring integers and discrete logarithms which can be solved in (quantum) poly-time thanks to QFS [31]. This does not contradict the existence of a (classical) poly-time algorithm solving GD. In this paper, we show how to exploit the algebraic structure of Goppa codes to construct a classical poly-time distinguisher with optimal advantage under some conditions on the rate of the code.

\footnotetext{
${ }^{3}$ Similarly to random codes, Goppa codes asymptotically meet the Gilbert-Varshamov bound. They have also a trivial permutation group like random codes.
} 


\section{Semantically Secure Conversions}

The fundamental issue when dealing with cryptographic primitives is to prove its security. A first approach is to show that the primitive resists to the best known attacks. However, this does not guarantee that there will not appear one day a better attack that renders the primitive insecure. The methodology of security proof by reduction addresses this question by linking a security notion that a cryptographic primitive should verify to an algorithmic problem widely considered as hard. The approach is similar to the one that proves the NP-Completeness of a given problem. Such a "security proof" proves that if an attacker exists then it can be used as a subroutine to solve a hard problem. In other words, such an attacker has little chances to exist.

These simple facts prompt to design conversions that would lead to an IND-CCA secure encryption scheme. The first article to propose such a conversion for the McEliece cryptosystem is [32] which proposes a conversion resulting into an IND-CCA2 in the Random Oracle Model under the assumption that the problem of decoding random linear codes is difficult. This work was then followed by [33] which proposes another modification while providing an IND-CPA secure encryption scheme in the standard model ${ }^{4}$ under the assumptions that both decoding random linear codes and distinguishing Goppa codes are difficult problems. Finally, under the same assumptions, [34] proposed (a modified) McEliece cryptosystem that is IND-CCA2 in the standard model.

\section{A Distinguisher of Alternant And Goppa Codes}

The McEliece cryptosystem relies on binary Goppa codes which belong to the class of alternant codes. We are now able to construct an algebraic system as explained in [19] for a key-recovey. This algebraic system will be the main ingredient for building a distinguisher. We assume that the public matrix is a $k \times n$ generator matrix $\boldsymbol{G}$ where by assumption $k=n-r m$ and such that it defines an alternant code of degree $r$. We know that the knowledge of a matrix $\boldsymbol{V}_{r}\left(\boldsymbol{x}^{*}, \boldsymbol{y}^{*}\right)$ for some vectors $\boldsymbol{x}^{*}$ and $\boldsymbol{y}^{*}$ allows to efficiently decode the public code defined by $\boldsymbol{G}$. Furthermore, from the definition of $\boldsymbol{G}$, we also know:

$$
\boldsymbol{V}_{r}\left(\boldsymbol{x}^{*}, \boldsymbol{y}^{*}\right) \boldsymbol{G}^{T}=\mathbf{0}
$$

Let $X_{1}, \ldots, X_{n}$ and $Y_{1}, \ldots, Y_{n}$ be $2 n$ variables corresponding to the $x_{i}^{*}$ 's and the $y_{i}^{*}$ 's. Observe that such $x_{i}^{*}$ 's and $y_{i}^{*}$ 's are a particular solution [19] of the following polynomial system:

$$
\bigcup_{e=0}^{r-1}\left\{\sum_{j=1}^{n} g_{i, j} Y_{j} X_{j}^{e}=0 \mid 1 \leqslant i \leqslant k\right\}
$$

where the $g_{i, j}$ 's are the entries of the known matrix $G$. Clearly, solving this system would lead to a possibly equivalent private key. For compact variants [35], [36] of McEliece [3], additional structures permit to drastically reduce the number of variables allowing to solve (2) for a large set of parameters in polynomial-time using dedicated Gröbner bases techniques [19]. But the general case is currently a major open question. However, we describe a simple way for partially solving (2). It basically consists in deriving a linear system from the polynomial system (2). Note that this operation is actually the first step performed during the computation of Gröbner bases algorithms such as by $F_{4}$ or $F_{5}$ [37], [38]. From now on, we will always assume that $q=2^{s}$ with $s \geqslant 1$. We can assume that $\boldsymbol{G}=\left(g_{i j}\right)$ with $1 \leqslant i \leqslant k$ and $1 \leqslant j \leqslant n$ is in reduced row echelon form over its $k$ first positions $\boldsymbol{G}=\left(\boldsymbol{I}_{k} \mid \boldsymbol{P}\right)$ where $\boldsymbol{P}=\left(p_{i j}\right)$ for $1 \leqslant i \leqslant k$ and $k+1 \leqslant j \leqslant n$ is the submatrix of $\boldsymbol{G}$ formed by its last $n-k=m r$ columns. Next, for any $i \in\{1, \ldots, k\}$ and $e \in\{0, \ldots, r-1\}$, we can rewrite (2) as $Y_{i} X_{i}^{e}=\sum_{j=k+1}^{n} p_{i, j} Y_{j} X_{j}^{e}$. Then, thanks to the trivial identity $Y_{i}\left(Y_{i} X_{i}^{2}\right)=\left(Y_{i} X_{i}\right)^{2}$ for all $i$ in $\{1, \ldots, k\}$, we get:

$$
\sum_{j=k+1}^{n} p_{i, j} Y_{j} \sum_{j=k+1}^{n} p_{i, j} Y_{j} X_{j}^{2}=\left(\sum_{j=k+1}^{n} p_{i, j} Y_{j} X_{j}\right)^{2} .
$$

We thus obtain a linear system $\mathcal{L}_{P}$ of $k$ equations involving $\left(\begin{array}{c}m r \\ 2\end{array}\right)$ variables $Z_{j j^{\prime}} \stackrel{\text { def }}{=} Y_{j} Y_{j^{\prime}} X_{j^{\prime}}^{2}+Y_{j^{\prime}} Y_{j} X_{j}^{2}$ which is as follows:

$$
\mathcal{L}_{\boldsymbol{P}} \stackrel{\text { def }}{=}\left\{\begin{array}{c}
\sum_{j=k+1}^{n-1} \sum_{j^{\prime}>j}^{n} p_{1, j} p_{i, j^{\prime}} Z_{j j^{\prime}}=0 \\
\vdots \\
\sum_{j=k+1}^{n-1} \sum_{j^{\prime}>j}^{n} p_{k, j} p_{i, j^{\prime}} Z_{j j^{\prime}}=0
\end{array}\right.
$$

Definition 6: For any integer $r \geqslant 1$ and $m \geqslant 1$, the number of variables $\left(\begin{array}{c}m r \\ 2\end{array}\right)$ in the linear system $\mathcal{L}_{\boldsymbol{P}}$ as defined in (3) is denoted by $N$ and its rank by $\operatorname{rank}\left(\mathcal{L}_{\boldsymbol{P}}\right)$. We denote by $\operatorname{Ker}\left(\mathcal{L}_{\boldsymbol{P}}\right)$ the kernel of $\mathcal{L}_{\boldsymbol{P}}$ and its dimension as a $\mathbb{F}_{q}$-vector space is denoted by $D$.

\footnotetext{
${ }^{4}$ There is no hash function in this model.
} 
Let us recall that $\operatorname{Ker}\left(\mathcal{L}_{\boldsymbol{P}}\right)$ is necessarily a $\mathbb{F}_{q}$-vector space since the linear system (3) have coefficients in $\mathbb{F}_{q}$ but the solutions of (2) are sought in the extension field $\mathbb{F}_{q^{m}}$. Furthermore, we obviously have $D=N-\operatorname{rank}\left(\mathcal{L}_{\boldsymbol{P}}\right)$. Hence, in order to recover the solutions of (2), it is necessary that $\operatorname{rank}\left(\mathcal{L}_{\boldsymbol{P}}\right)$ is almost equal to the number of variables $N=\left(\begin{array}{c}m r \\ 2\end{array}\right)$. For a random system, this is likely to happen when the number $k$ of equations in (3) is greater than the number of unknowns, that is to say $k \geqslant N$. It appears experimentally that $D$ is amazingly large even in the case where $k \geqslant N$. It even depends on whether or not the code with generator matrix $\boldsymbol{G}$ is chosen as a (generic) alternant code or as a Goppa code. Interestingly enough, when $\boldsymbol{G}$ is chosen at random, $\operatorname{rank}\left(\mathcal{L}_{\boldsymbol{P}}\right)$ is equal to $\min \{k, N\}$ with very high probability. In particular, the dimension of the solution space is typically 0 when $k$ is larger than the number of variables $N$ as one would expect. This will be proved in Section VI. Although this defect in the rank is an obstacle to break the McEliece cryptosystem, it can be used to distinguish the public generator of a structured code from a random code.

We consider three cases. First, when the $p_{i j}$ 's are chosen uniformly and independently at random in $\mathbb{F}_{q}$ then we denote by $D_{\text {random }}$ the dimension of $\operatorname{Ker}\left(\mathcal{L}_{\boldsymbol{P}}\right)$. When $\boldsymbol{G}$ is chosen as a generator matrix of a random alternant (resp. Goppa) code of degree $r$, we denote it by $D_{\text {alternant }}$ (resp. $D_{\text {Goppa }}$ ). We carried out intensive computations with Magma [39] by randomly generating alternant and Goppa codes over the field $\mathbb{F}_{q}$ with $q \in\{2,4,8,16,32\}$ for $r$ in the range $\{3, \ldots, 50\}$ and several values of $m$. Furthermore, in our probabilistic model, a random alternant code is obtained by picking uniformly and independently at random two vectors $\left(x_{1}, \ldots, x_{n}\right)$ and $\left(y_{1}, \ldots, y_{n}\right)$ from $\left(\mathbb{F}_{q^{m}}\right)^{n}$ such that the $x_{i}$ 's are all different and the $y_{i}$ 's are all nonzero. A random Goppa code is obtained by taking a random vector $\left(x_{1}, \ldots, x_{n}\right)$ in $\left(\mathbb{F}_{q^{m}}\right)^{n}$ with all the $x_{i}$ 's different and a random irreducible polynomial $\gamma(z)=\sum_{i} \gamma_{i} z^{i}$ of degree $r$. Our experiments have revealed that the dimension of $\operatorname{Ker}\left(\mathcal{L}_{\boldsymbol{P}}\right)$ is predictable and follows formulas.

Experimental Fact 1 (Alternant Case): As long as $N-D_{\text {alternant }}<k, D_{\text {alternant }}$ is equal with high probability to:

$$
T_{\text {alternant }} \stackrel{\text { def }}{=} \frac{1}{2} m(r-1)\left((2 e+1) r-2 \frac{q^{e+1}-1}{q-1}\right)
$$

with $e \stackrel{\text { def }}{=}\left\lfloor\log _{q}(r-1)\right\rfloor$.

Experimental Fact 2 (Goppa Case): As long as $N-D_{\mathrm{Goppa}}<k$ then $D_{\mathrm{Goppa}}$ is equal with high probability to $T_{\mathrm{Goppa}}$ which is defined when $r<q-1$ as:

$$
T_{\text {Goppa }} \stackrel{\text { def }}{=} \frac{1}{2} m(r-1)(r-2)=T_{\text {alternant }}
$$

and when $r \geqslant q-1$ :

$$
T_{\mathrm{Goppa}} \stackrel{\text { def }}{=} \frac{1}{2} m r\left((2 e+1) r-2(q-1) q^{e-1}-1\right)
$$

with $e$ being the unique integer such that:

$$
(q-1)^{2} q^{e-2}<r \leqslant(q-1)^{2} q^{e-1} .
$$

We gathered in Appendix A some experimental results obtained through intensive computations with the Magma system [39].

\section{Alternant CAse}

The goal of this section is to explain the value of the dimension $D_{\text {alternant }}$ of $\operatorname{Ker}\left(\mathcal{L}_{\boldsymbol{P}}\right)$ for $q$-ary alternant codes of degree $r$. We shall see that this dimension will be obtained by first identifying a $\mathbb{F}_{q^{m}}$-basis of $\operatorname{Ker}\left(\mathcal{L}_{\boldsymbol{P}}\right)$ when viewed as a linear system with coefficients in $\mathbb{F}_{q^{m}}$. To set up the linear system $\mathcal{L}_{\boldsymbol{P}}$ as defined in (3), we have used the trivial identity $Y_{i} Y_{i} X_{i}^{2}=\left(Y_{i} X_{i}\right)^{2}$. The fundamental remark is that we can use any identity $Y_{i} X_{i}^{a} Y_{i} X_{i}^{b}=Y_{i} X_{i}^{c} Y_{i} X_{i}^{d}$ with $a, b, c, d \in\{0,1, \ldots, r-1\}$ such that $a+b=c+d$. Such identities lead to the same algebraic system $\mathcal{L}_{\boldsymbol{P}}$ :

$$
\begin{aligned}
\sum_{\left(j, j^{\prime}\right) \in J} p_{i, j} p_{i, j^{\prime}} & \left(Y_{j} X_{j}^{a} Y_{j^{\prime}} X_{j^{\prime}}^{b}+Y_{j^{\prime}} X_{j^{\prime}}^{a} Y_{j} X_{j}^{b}\right. \\
& \left.+Y_{j} X_{j}^{c} Y_{j^{\prime}} X_{j^{\prime}}^{d}+Y_{j^{\prime}} X_{j^{\prime}}^{c} Y_{j} X_{j}^{d}\right)=0
\end{aligned}
$$

where we have set :

$$
J \stackrel{\text { def }}{=}\left\{\left(j, j^{\prime}\right) \in \mathbb{N} \times \mathbb{N} \mid k+1 \leqslant j<j^{\prime} \leqslant n\right\} .
$$

The fact that there are many different ways of combining equations together yielding the same linear system $\mathcal{L}_{\boldsymbol{P}}$ explains why the dimension of $\operatorname{Ker}\left(\mathcal{L}_{\boldsymbol{P}}\right)$ is large. In what follows, we exhibit further elements of $\operatorname{Ker}\left(\mathcal{L}_{\boldsymbol{P}}\right)$ thanks to the automorphisms $x \longmapsto x^{q^{\ell}}$ where $\ell$ is in $\{0, \ldots, m-1\}$. Indeed, we can also consider the identity:

$$
\left(Y_{i} X_{i}^{a}\right)^{q^{\ell^{\prime}}}\left(Y_{i} X_{i}^{b}\right)^{q^{\ell}}=\left(Y_{i} X_{i}^{c}\right)^{q^{\ell^{\prime}}}\left(Y_{i} X_{i}^{d}\right)^{q^{\ell}}
$$


for any integers $a, b, c, d, \ell$ and $\ell^{\prime}$ such that $a q^{\ell^{\prime}}+b q^{\ell}=c q^{\ell^{\prime}}+d q^{\ell}$. We get again the linear system $\mathcal{L}_{\boldsymbol{P}}$. However, assuming that $\ell^{\prime} \leqslant \ell$, solutions obtained from such equations are exactly those coming from the identity:

$$
Y_{i} X_{i}^{a} Y_{i}^{q^{\ell-\ell^{\prime}}} X_{i}^{b q^{\ell-\ell^{\prime}}}=Y_{i} X_{i}^{c} Y_{i}^{q^{\ell-\ell^{\prime}}} X_{i}^{d q^{\ell-\ell^{\prime}}} .
$$

We can focus on vectors that satisfy equations obtained with $0 \leqslant a, b, c, d<r, 0 \leqslant \ell<m$ and $a+q^{\ell} b=c+q^{\ell} d$. Without loss of generality, we can assume that $d>b$ and let us set $\delta=d-b$. Moreover, the equality $a+q^{\ell} b=c+q^{\ell} d$ implies that $a=c+q^{\ell} \delta$.

We now try to determine the number of linearly independent solutions induced by such identities. For the sake of simplicity, we denote by $\boldsymbol{Z}=\left(Z_{j, j^{\prime}}\right)_{\left(j, j^{\prime}\right) \in J}$ the vector that is obtained from the identity $a+q^{\ell} b=c+q^{\ell} d$. One can show that:

$$
Z_{j, j^{\prime}}=\left(X_{j}^{\delta}+X_{j^{\prime}}^{\delta}\right)^{q^{\ell}}\left(Y_{j} Y_{j^{\prime}}^{q^{\ell}} X_{j}^{c} X_{j^{\prime}}^{q^{\ell} b}+Y_{j^{\prime}} Y_{j}^{q^{\ell}} X_{j^{\prime}}^{c} X_{j}^{q^{\ell} b}\right) .
$$

Thus any solution obtained by the tuple $(a, b, c, d, \ell)$ is uniquely described by $(b, c, \delta, \ell)$ by setting $d=b+\delta$ and $a=c+q^{\ell} \delta$ provided that $1 \leqslant \delta \leqslant r-1-b, 0 \leqslant b \leqslant r-2$ and $0 \leqslant c+q^{\ell} \delta \leqslant r-1$. In the sequel, we set $e \stackrel{\text { def }}{=}\left\lfloor\log _{q}(r-1)\right\rfloor$ so that $0 \leqslant \ell \leqslant e$. We will show that $\boldsymbol{Z}$ can be expressed as a linear combination of some solutions obtained thanks to very specific identities. Firstly, let us denote by $\boldsymbol{B}(b, c, \ell)$ the solution obtained form $(b, c, \delta, \ell)$ with $\delta=1$. We define:

$$
\mathcal{B}_{r} \stackrel{\text { def }}{=} \bigcup_{\substack{0 \leqslant b \leqslant r-2 \\ 0 \leqslant \ell \leqslant e}}\left\{\boldsymbol{B}(b, c, \ell) \neq \mathbf{0} \mid 0 \leqslant c \leqslant r-1-q^{\ell}\right\} .
$$

It is easy to see that if $\boldsymbol{B}(b, c, 0)$ belongs to $\mathcal{B}_{r}$ then $0 \leqslant b<c \leqslant r-2$. We denote by $<\mathcal{B}_{r}>$ the vector space spanned by $\mathcal{B}_{r}$. We are now in position to show that $Z$ belongs to $\left\langle\mathcal{B}_{r}\right\rangle$. First notice from Equation (7) that if $\boldsymbol{W}$ is the solution obtained from $a+q^{\ell} b=g+q^{\ell} f$ for some integers $g$ and $f$ then $\boldsymbol{Z}+\boldsymbol{W}$ is the vector that would be obtained from $c+q^{\ell} d=g+q^{\ell} f$. In particular, the identity $c+q^{\ell} \delta+b q^{\ell}=c+q^{\ell}(b+\delta)$ can also be rewritten as $c+q^{\ell} \delta+b q^{\ell}=c+q^{\ell}(\delta-1)+(b+1) q^{\ell}$. Therefore, we see that $\boldsymbol{Z}=\boldsymbol{B}\left(b, c+q^{\ell}(\delta-1), \ell\right)+\boldsymbol{V}$ where $\boldsymbol{V}$ is the solution obtained from the identity

$$
c+q^{\ell}(\delta-1)+(b+1) q^{\ell}=c+q^{\ell}(b+\delta) .
$$

Hence by induction on $\delta$ we can prove that:

$$
\boldsymbol{Z}=\sum_{i=1}^{\delta} \boldsymbol{B}\left(b+i-1, c+q^{\ell}(\delta-i), \ell\right) .
$$

Proposition 2: For all $r \geqslant 3$, let us denote by $\left|\mathcal{B}_{r}\right|$ the cardinality of $\mathcal{B}_{r}$ then:

$$
T_{\text {alternant }}=m\left|\mathcal{B}_{r}\right| \text {. }
$$

Proof: The number of elements in $\mathcal{B}_{r}$ is given by the number of possible tuples $(b, c, \ell)$ that is to say:

$$
\begin{aligned}
\left|\mathcal{B}_{r}\right| & =\frac{1}{2}(r-1)(r-2)+\sum_{\ell=1}^{e} \sum_{b=0}^{r-2}\left(r-q^{\ell}\right) \\
& =\frac{1}{2}(r-1)\left(r-2+2 e r-2 \sum_{\ell=1}^{e} q^{\ell}\right)=\frac{1}{m} T_{\text {alternant }} .
\end{aligned}
$$

This proposition shows that $\mathcal{B}_{r}$ is a $\mathbb{F}_{q^{m}}$-basis that provides a $\mathbb{F}_{q}$-basis of $\operatorname{Ker}\left(\mathcal{L}_{\boldsymbol{P}}\right)$ allowing us to suggest an heuristic: consider an arbitrary decomposition of the elements of $\mathbb{F}_{q^{m}}$ in a $\mathbb{F}_{q}$ basis. Let $\pi_{i}: \mathbb{F}_{q^{m}} \rightarrow \mathbb{F}_{q}$ be the function giving the $i$-th coordinate in this decomposition with $1 \leqslant i \leqslant m$. By extension we denote for $\boldsymbol{z}=\left(z_{j}\right)_{1 \leqslant j \leqslant n} \in\left(\mathbb{F}_{q^{m}}\right)^{n}$ by $\pi_{i}(\boldsymbol{z})$ the vector $\left(\pi_{i}\left(z_{j}\right)\right)_{1 \leqslant j \leqslant n} \in \mathbb{F}_{q}^{n}$.

Heuristic 1: For any $j$ such that $1 \leqslant j \leqslant n$ and for random choices of $x_{j}$ 's and $y_{j}$ 's then $\bigcup_{1 \leqslant i \leqslant m}\left\{\pi_{i}(\boldsymbol{Z}) \mid \boldsymbol{Z} \in \mathcal{B}_{r}\right\}$ forms a basis of $\operatorname{Ker}\left(\mathcal{L}_{P}\right)$.

\section{Binary Goppa CASE}

In this section, we will investigate the case of binary Goppa codes. Notice that for $q$-ary Goppa codes of degree $r<q-1$ we have observed that $T_{\text {alternant }}=T_{\text {Goppa }}$ because (4) simplifies to:

$$
\frac{1}{2} m(r-1)(r-2) \stackrel{\text { def }}{=} T_{\text {Goppa }} .
$$


This is due to the fact that $e=0$ when $r<q-1$. We leave as an open question the proof that $q$-ary Goppa codes of degree $r<q-1$ behave for our distinguisher as alternant codes. We focus on the classical case in code-based cryptography of binary Goppa codes.

The goal is to identify a basis of $\operatorname{Ker}\left(\mathcal{L}_{\boldsymbol{P}}\right)$ for binary Goppa codes of degree $r$. We assume therefore that $q=2$. In that special case, the theoretical expression $T_{\text {Goppa }}$ (Experimental Fact 2) has a simpler expression.

Proposition 3: Let $e=\left\lceil\log _{2} r\right\rceil+1$. When $q=2$ then (6) can be simplified to:

$$
T_{\mathrm{Goppa}}=\frac{1}{2} m r\left((2 e+1) r-2^{e}-1\right) .
$$

Theorem 1 shows that a binary Goppa code of degree $r$ can be regarded as a binary alternant code of degree $2 r$. This seems to indicate that we should have

$$
D_{\text {Goppa }}(r)=T_{\text {alternant }}(2 r) \text {. }
$$

This is not the case though because it turns out that $D_{\mathrm{Goppa}}(r)$ is significantly smaller than this. In our experiments, we have found out that the vectors of $\mathcal{B}_{2 r}$ still form a generating set for $\operatorname{Ker}\left(\mathcal{L}_{P}\right)$. Unfortunately, they are not independent anymore. Our goal is therefore to identify the additional dependencies occuring in $\mathcal{B}_{2 r}$. We will see that many of them come from $\mathbb{F}_{2^{m}}$-relations induced by the Goppa polynomial $\gamma(z)=\sum_{i=0}^{r} \gamma_{i} z^{i}$ with $\gamma_{r} \neq 0$. Recall that by definition $Y_{i}=\gamma\left(X_{i}\right)^{-2}$. This fact will allow to derive two types of linear dependencies. The first type of linear relations is rather natural, whilst the second type is more subtle. In the sequel we set $u \stackrel{\text { def }}{=}\left\lfloor\log _{2}(2 r-1)\right\rfloor$.

\section{A. Linear Dependencies over $\mathbb{F}_{2^{m}}$}

We derive a first set of linear dependencies induced by the Goppa polynomial $\gamma(X)$.

Proposition 4: Let $t, \ell$ and $c$ be such that $0 \leqslant t \leqslant r-2,1 \leqslant \ell \leqslant u$ and $0 \leqslant c \leqslant 2 r-2^{\ell}-1$ then it holds:

$$
\begin{aligned}
& \sum_{b=0}^{r} \gamma_{b}^{2^{\ell}} \boldsymbol{B}(t+b, c, \ell)= \\
& \boldsymbol{B}\left(2 t, c+2^{\ell-1}, \ell-1\right)+\boldsymbol{B}(2 t+1, c, \ell-1) .
\end{aligned}
$$

Proof: Let us set $\boldsymbol{V}=\left(V_{i, j^{\prime}}\right)_{\left(i, j^{\prime}\right) \in J}$ as the following:

$$
\boldsymbol{V} \stackrel{\text { def }}{=} \sum_{b=0}^{r} \gamma_{b}^{2^{\ell}} \boldsymbol{B}(t+b, c, \ell)
$$

The equality $Y_{j} \gamma\left(X_{j}\right)^{2}=1$ clearly implies that $\sum_{b=0}^{r} \gamma_{b}^{2} Y_{j}^{2} X_{j}^{2(b+t)}=Y_{j} X_{j}^{2 t}$ and therefore:

$$
V_{j, j^{\prime}}=\left(X_{j}+X_{j^{\prime}}\right)^{2^{\ell}}\left(Y_{j} Y_{j^{\prime}}^{2^{\ell-1}} X_{j}^{c} X_{j^{\prime}}^{2^{\ell} t}+Y_{j^{\prime}} Y_{j}^{2^{\ell-1}} X_{j^{\prime}}^{c} X_{j}^{2^{\ell} t}\right) .
$$

One can check that $\boldsymbol{V}$ is exactly the vector that we would obtain from the identity $a^{*}+2^{\ell-1} b^{*}=c+2^{\ell-1}\left(b^{*}+2\right)$ with $a^{*} \stackrel{\text { def }}{=} c+2^{\ell}$ and $b^{*} \stackrel{\text { def }}{=} 2 t$. Hence by (10) the vector $\boldsymbol{V}$ can be written as:

$$
\boldsymbol{V}=\boldsymbol{B}\left(2 t, c+2^{\ell-1}, \ell-1\right)+\boldsymbol{B}(2 t+1, c, \ell-1) .
$$

This last equality terminates the proof.

Consequently $\mathcal{B}_{2 r}$ cannot provide a basis for $\operatorname{Ker}\left(\mathcal{L}_{\boldsymbol{P}}\right)$ as in the alternant case. It even possible to count the number of linear dependencies predicted by Proposition 4. Indeed, each equation is defined by a unique $(t, c, \ell)$ such that $0 \leqslant t \leqslant r-2,1 \leqslant \ell \leqslant u$ and $0 \leqslant c \leqslant 2 r-2^{\ell}-1$. So if $N_{L}$ is the number of equations of the form (11) then clearly we have:

$$
N_{L}=2(r-1)\left(r u+1-2^{u}\right) .
$$

\section{B. Additional Relations Inducing Dependencies over $\mathbb{F}_{2}$}

We exhibit new linear dependencies between some elements of $\mathcal{B}_{2 r}$ and the vectors $\boldsymbol{B}^{2}(b, c, \ell)$.

Proposition 5: For any $\ell, b$ and $t$ such that $0 \leqslant \ell \leqslant u-1,0 \leqslant b \leqslant 2 r-2$ and $0 \leqslant t \leqslant r-1-2^{\ell}$, we have:

$$
\boldsymbol{B}(b, 2 t, \ell+1)=\sum_{c=0}^{r} \gamma_{c}^{2} \boldsymbol{B}^{2}(b, t+c, \ell) .
$$

Proof: Let us set $\boldsymbol{V}=\left(V_{i, j^{\prime}}\right)_{\left(i, j^{\prime}\right) \in J}$ as the vector:

$$
\boldsymbol{V} \stackrel{\text { def }}{=} \sum_{c=0}^{r} \gamma_{c}^{2} \boldsymbol{B}^{2}(b, t+c, \ell) .
$$


On can see that for all $\left(j, j^{\prime}\right) \in J$ :

$$
\begin{aligned}
V_{j, j^{\prime}}=\left(X_{j}+X_{j^{\prime}}\right)^{2^{\ell+1}} & \left(Y_{j} Y_{j^{\prime}}^{2^{\ell+1}} X_{j}^{2 t} X_{j^{\prime}}^{2^{\ell+1} b}\right. \\
& \left.+Y_{j^{\prime}} Y_{j}^{2^{\ell+1}} X_{j^{\prime}}^{2 t} X_{j}^{2^{\ell+1} b}\right) .
\end{aligned}
$$

We recognize that $\boldsymbol{V}$ is exactly the expression of $\boldsymbol{B}(b, 2 t, \ell+1)$ and hence the proposition is proved.

We can count the number of linearly dependencies predicted by Proposition 5 . Let $N_{Q}$ be the number of vectors of $\mathcal{B}_{2 r}$ satisfying Equation (13). By Proposition $5 N_{Q}$ is exactly the number of $(\ell, b, t)$ so that:

$$
N_{Q}=(2 r-1)\left(r u-2^{u}+1\right) \text {. }
$$

\section{Counting the Exact Number of Linear Dependencies over $\mathbb{F}_{2}$}

We now want to count the number of linear dependencies induced by Proposition 5 and Proposition 4. The difficulty is that some of the $N_{Q}$ vectors of $\mathcal{B}_{2 r}$ are counted twice because they appear both in linear relations of the form (11) and "quadratic" equations of the form (13). Let $N_{L \cap Q}$ be the number of such vectors. More precisely, let $\mathcal{Q}_{2 r}$ be the subset of vectors $\boldsymbol{B}(b, c, \ell)$ of $\mathcal{B}_{2 r}$ which are involved in an Equation of type (13). Remark in that case $c$ has to be even and $\ell \geqslant 1$. Furthermore, there are equations of type (11) which involve only vectors of $\mathcal{Q}_{2 r}$. Let $N_{1}$ be their number. Moreover, it is possible by adding two equations of type (11) involving at least one vector which is not in $\mathcal{Q}_{2 r}$ to obtain an equation which involves only vectors of $\mathcal{Q}_{2 r}$. Let $N_{0}$ be the number of such sums and let $N_{L \cap Q} \stackrel{\text { def }}{=} N_{1}+N_{0}$. Our goal is to prove that:

$$
N_{L \cap Q}=(r-1)\left(\left(u-\frac{1}{2}\right) r-2^{u}+2\right) .
$$

Proof: We will consider vectors of $\mathcal{Q}_{2 r}$, that is to say vectors of $\mathcal{B}_{2 r}$ that satisfy Equation (13), such that there exists a linear relation that link them. In other words, we consider all the linear relations of the form:

$$
\sum_{i} \alpha_{i} \boldsymbol{B}\left(b_{i}, c_{i}, \ell_{i}\right)=0
$$

with $\alpha_{i}$ in $\mathbb{F}_{2^{m}}$ and where each $\boldsymbol{B}\left(b_{i}, c_{i}, \ell_{i}\right)$ is equal to a linear relation of the form (13).

One can observe that for such vectors we necessarily have $c_{i}$ even and $1 \leqslant \ell_{i} \leqslant u$. In particular, an equation of the form of Equation (11) will involve only vectors of $\mathcal{Q}_{2 r}$ if and only if $\ell-1 \geqslant 1$ and $c$ is even. Let us recall that the number of such equations is $N_{1}$, and consequently, its value is $\sum_{t=0}^{r-2} \sum_{\ell=2}^{u} \frac{1}{2}\left(2 r-2^{\ell}\right)$, namely:

$$
N_{1}=(r-1)\left((u-1) r-2^{u}+2\right) \text {. }
$$

On the other hand when $\ell=1$ and $c$ is even, say for instance $c=2 t^{\prime}$ then Equation (11) becomes:

$$
\sum_{b=0}^{r} \gamma_{b}^{2} \boldsymbol{B}\left(t+b, 2 t^{\prime}, 1\right)=\boldsymbol{B}\left(2 t, 2 t^{\prime}+1,0\right)+\boldsymbol{B}\left(2 t+1,2 t^{\prime}, 0\right) \text {. }
$$

Notice that we always have $\boldsymbol{B}(b, c, 0)=\boldsymbol{B}(c, b, 0)$. So if $t^{\prime}=t$ then $\boldsymbol{B}(2 t, 2 t+1,0)+\boldsymbol{B}(2 t+1,2 t, 0)=\mathbf{0}$ and when $t \neq t^{\prime}$ we obviously have:

$$
\sum_{b=0}^{r} \gamma_{b}^{2} \boldsymbol{B}\left(t+b, 2 t^{\prime}, 1\right)=\sum_{b=0}^{r} \gamma_{b}^{2} \boldsymbol{B}\left(t^{\prime}+b, 2 t, 1\right) .
$$

In both case, we get new linear dependencies between vectors of $\mathcal{Q}_{2 r}$ different from those already obtained.In conclusion, $N_{0}$ is exactly the number of sets $\left\{t, t^{\prime}\right\}$. By assumption $t$ and $t^{\prime}$ have to satisfy $0 \leqslant t \leqslant r-2$ and $c=2 t^{\prime}$ with $0 \leqslant c \leqslant 2 r-3$, which implies that $0 \leqslant t^{\prime} \leqslant r-2$. $N_{0}$ is therefore the number of couples $\left(t, t^{\prime}\right)$ such that $0 \leqslant t \leqslant t^{\prime} \leqslant r-2$. By gathering all the cases we have proved that:

$$
N_{L \cap Q}=(r-1)\left((u-1) r-2^{u}+2\right)+\frac{1}{2}(r-1) r .
$$

Proposition 6: For any integer $r \geqslant 2$, we have:

$$
\begin{aligned}
\frac{1}{m} T_{\text {Goppa }}(r) & =\left|\mathcal{B}_{2 r}\right|-N_{L}-N_{Q}+N_{L \cap Q} \\
& =\frac{1}{2} r\left((2 u+3) r-2^{u+1}-1\right) .
\end{aligned}
$$

Proof: From Equation (4) and gathering all the equalities:

$$
\left|\mathcal{B}_{2 r}\right|-\left(N_{L}+N_{Q}-N_{L \cap Q}\right)=r\left(\left(u+\frac{3}{2}\right) r-2^{u}-\frac{1}{2}\right) .
$$


Moreover, Proposition 3 gives:

$$
\frac{1}{m} T_{\text {Goppa }}(r)=\frac{1}{2} r\left((2 e+1) r-2^{e}-1\right)
$$

where $e=\left\lceil\log _{2} r\right\rceil+1$. Using the basic inequality $2 r-1<2 r<2(2 r-1)$, we have therefore $\log _{2}(2 r-1)<\log _{2}(r)+1<$ $\log _{2}(2 r-1)+1$. This implies $\left\lceil\log _{2} r\right\rceil=u$ and thus $\frac{1}{m} T_{\mathrm{Goppa}}(r)=\frac{1}{2} r\left((2 u+3) r-2^{u+1}-1\right)$.

\section{RANDOM CASE}

The purpose of this section is to study the behavior of $D_{\text {random }}$, namely the dimension of $\operatorname{Ker}\left(\mathcal{L}_{\boldsymbol{P}}\right)$ as $\mathbb{F}_{q}$-vector space when the entries of the matrix $P$ are drawn independently from the uniform distribution over $\mathbb{F}_{q}$. In this case, we can show that:

Theorem 2: Assume that $N \leqslant k$ and that the entries of $P$ are drawn independently from the uniform distribution over $\mathbb{F}_{q}$. Then for any function $\omega(x)$ tending to infinity as $x$ goes to infinity, we have

$$
\operatorname{Pr}\left(D_{\text {random }} \geqslant m r \omega(m r)\right)=o(1)
$$

as $m r$ goes to infinity.

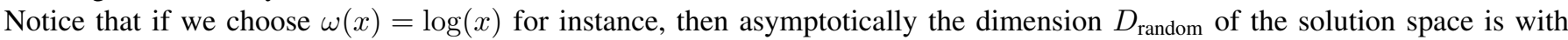
very large probability smaller than $m r \log (m r)$. When $m$ and $r$ are of the same order (which is generally chosen in practice) this quantity is smaller than $D_{\text {alternant }}$ or $D_{\text {Goppa }}$ which are of the form $\Omega\left(m r^{2}\right)$.

The main ingredient for proving Theorem 2 consists in analyzing a certain (partial) Gaussian elimination process on the matrix:

$$
M \stackrel{\text { def }}{=}\left(p_{i j} p_{i j^{\prime}}\right) \underset{\substack{1 \leqslant i \leqslant k \\ k+1 \leqslant j<j^{\prime} \leqslant n}}{1} .
$$

We can see the matrix $M$ in block form, each block consists of the matrix:

$$
B_{j}=\left(p_{i, k+j} p_{i, k+j^{\prime}}\right) \underset{\substack{1 \leqslant i \leqslant k \\ 1 \leqslant j<j^{\prime} \leqslant n-k}}{ } .
$$

Each block $B_{j}$ is of size $k \times(r m-j)$. Notice that in $B_{j}$, the rows for which $p_{i, k+j}=0$ consist only of zeros. To start the Gaussian elimination process with $B_{1}$, we will therefore choose $r m-1$ rows for which $p_{i, k+1} \neq 0$. This gives a square matrix $M_{1}$. We perform Gaussian elimination on $M$ by adding rows involved in $M_{1}$ to put the first block $B_{1}$ in standard form. We continue this process with $B_{2}$ by picking now $r m-2$ rows which have not been chosen before and which correspond to $p_{i, k+2} \neq 0$. This yields a square submatrix $M_{2}$ of size $r m-2$ and we continue this process until we reach the last block. The key observation is that:

$$
\operatorname{rank}(M) \geqslant \operatorname{rank}\left(M_{1}\right)+\cdots+\operatorname{rank}\left(M_{r m-1}\right) .
$$

A rough analysis of this process yields Theorem 2 . The important point is that what happens for different blocks are independent processes and it corresponds to looking at different rows of the matrix $P$. We give all the previous results that we need in order to prove Theorem 2 .

It will be convenient to assume that the columns of $M$ are ordered lexicographically. The index of the first column is $\left(j, j^{\prime}\right)=(k+1, k+2)$, the second one is $\left(j, j^{\prime}\right)=(k+1, k+3)$, while the last one is $\left(j, j^{\prime}\right)=(n-1, n)$. The matrices $M_{i}$ 's which are involved in the Gaussian elimination process mentioned above are defined inductively as follows. Let $E_{1}$ be the subset of $\{1, \ldots, k\}$ of indices $s$ such that $p_{s, k+1} \neq 0$. Let $F_{1}$ be the subset of $E_{1}$ formed by its first $r m-1$ elements (if these elements exist). Now, we set

$$
M_{1} \stackrel{\text { def }}{=}\left(p_{s, k+1} p_{s, j}\right) \underset{\substack{s \in F_{1} \\ k+1<j \leqslant n}}{ } .
$$

Let $r_{1}$ be the rank of $M_{1}$. To simplify the discussion, we assume that:

1) $F_{1}=\{1,2, \ldots, r m-1\}$,

2) the submatrix $N_{1}$ of $M_{1}$ formed by its first $r_{1}$ rows and columns is of full rank.

Note that we can always assume this by performing suitable row and column permutations. In other words $M$ has the following block structure:

$$
M=\left(\begin{array}{ll}
N_{1} & B_{1} \\
A_{1} & C_{1}
\end{array}\right) .
$$

We denote:

$$
M^{(1)} \stackrel{\text { def }}{=}\left(\begin{array}{cc}
N_{1}^{-1} & O \\
-A_{1} N_{1}^{-1} & I
\end{array}\right) M,
$$

where $O$ is a matrix of size $r_{1} \times\left(k-r_{1}\right)$ with only zero entries and $I$ is the identity matrix of size $k-r_{1}$. Notice that $M^{(1)}$ takes the block form:

$$
M^{(1)}=\left(\begin{array}{cc}
I & B_{1}^{\prime} \\
O & C_{1}^{\prime}
\end{array}\right) .
$$


This is basically performing Gaussian elimination on $M$ in order to have the first $r_{1}$ columns in standard form. We then define inductively the $E_{i}, F_{i}, M_{i}, M^{(i)}$ and $N_{i}$ as follows:

$$
\begin{aligned}
& E_{i} \stackrel{\text { def }}{=}\left\{s \mid 1 \leqslant s \leqslant k, p_{s, k+i} \neq 0\right\} \backslash \bigcup_{u=1}^{i-1} F_{i-u}, \\
& F_{i} \stackrel{\text { def }}{=} \text { the first } r m-i \text { elements of } E_{i} .
\end{aligned}
$$

$M_{i}$ is the submatrix of $M^{(i-1)}$ obtained from the rows in $F_{i}$ and the columns associated to the indices of the form $\left(k+i, j^{\prime}\right)$ where $j^{\prime}$ ranges from $k+i+1$ to $n . M^{(i)}$ is obtained from $M^{(i-1)}$ by first choosing a square submatrix $N_{i}$ of $M_{i}$ of full rank and with the same rank as $M_{i}$ and then by performing Gaussian elimination on the rows in order to put the columns of $M^{(i-1)}$ involved in $N_{i}$ in standard form (i.e., the submatrix of $M^{(i-1)}$ corresponding to $N_{i}$ becomes the identity matrix while the other entries in the columns involved in $N_{i}$ become zero). It is clear that the whole process leading to $M^{(r m-1)}$ amounts to perform (partial) Gaussian elimination to $M$. Hence:

Lemma 1: When $\left|E_{i}\right| \geqslant r m-i$, for all $i \in\{1, \ldots, r m-1\}$, we have:

$$
\operatorname{rank}(M) \geqslant \sum_{i=1}^{r m-1} \operatorname{rank}\left(M_{i}\right) \text {. }
$$

Another observation is that $M_{i}$ is equal to the sum of the submatrix $\left(p_{s, k+i} p_{s, j}\right)_{\substack{s \in F_{i} \\ k+i<j \leqslant n}}$ of $M$ and a certain matrix which is some function on the entries $p_{t, k+i} p_{t, j}$ where $t$ belongs to $F_{1} \cup \ldots F_{i-1}$ and $j$ ranges over $\{k+i+1, n\}$. Since by definition of $F_{i}, p_{s, k+i}$ is different from 0 for $s$ in $F_{i}$. In addition, the rank of $M_{i}$ does not change by multiplying each row of index $s$ by $p_{s, k+i}^{-1}$. Then, it turns out that the rank of $M_{i}$ is equal to the rank of a matrix which is the sum of the matrix $\left(p_{s, j}\right)_{k+i<j \leqslant n}$, another matrix depending on the $p_{t, k+i} p_{t, j}$ 's (where $t$ ranges over $F_{1} \cup \ldots F_{i-1}$ ) and the $p_{s, k+1}$ 's with $s \in F_{i}$. This proves that:

Lemma 2: Assume that $\left|E_{i}\right| \geqslant r m-i$ for all $i \in\{1, \ldots, r m-1\}$. Then, the random variables $r a n k\left(M_{i}\right)$ are independent and $\operatorname{rank}\left(M_{i}\right)$ is distributed as the rank of a square matrix of size $r m-i$ with entries drawn independently from the uniform distribution on $\mathbb{F}_{q}$.

Another essential ingredient for proving Theorem 2 is the following well known lemma (see for instance [40][Theorem 1])

Lemma 3: There exist two positive constants $A$ and $B$ depending on $q$ such that the probability $p(s, \ell)$ that a random $\ell \times \ell$ matrix over $\mathbb{F}_{q}$ is of rank $\ell-s$ (where the coefficients are drawn independently from each other from the uniform distribution on $\mathbb{F}_{q}$ ) satisfies

$$
\frac{A}{q^{s^{2}}} \leqslant p(s, \ell) \leqslant \frac{B}{q^{s^{2}}} .
$$

This enables to control the exponential moments of the defect of a random matrix.For a square matrix $M$ of size $\ell \times \ell$, we define the defect $d(M)$ by $d(M) \stackrel{\text { def }}{=} \ell-\operatorname{rank}(M)$.

Lemma 4: If $M$ is random square matrix whose entries are drawn independently from the uniform distribution over $\mathbb{F}_{q}$, then there exists some constant $K$ such that for every $\lambda>0$,

$$
\mathbb{E}\left(q^{\lambda d(M)}\right) \leqslant K q^{\frac{\lambda^{2}}{4}}
$$

$\mathbb{E}($.$) denoting the expectation.$

Proof: By using Lemma 3, we obtain:

$$
\mathbb{E}\left(q^{\lambda d(M)}\right) \leqslant \sum_{d=0}^{\infty} q^{\lambda d} \frac{B}{q^{d^{2}}} \leqslant B \sum_{d=0}^{\infty} q^{\lambda d-d^{2}}
$$

Observe that the maximum of the function $d \mapsto q^{\lambda d-d^{2}}$ is reached for $d_{0}=\frac{\lambda}{2}$ and is equal to $q^{\frac{\lambda^{2}}{4}}$. Then, we can write the sum above as:

$$
\sum_{d=0}^{\infty} q^{\lambda d-d^{2}}=\sum_{d \leqslant d_{0}} q^{\lambda d-d^{2}}+\sum_{d>d_{0}} q^{\lambda d-d^{2}}
$$

Finally, we notice that:

$$
\begin{aligned}
& \frac{q^{\lambda(d+1)-(d+1)^{2}}}{q^{\lambda d-d^{2}}} \leqslant \frac{q^{\lambda\left(d_{0}+1\right)-\left(d_{0}+1\right)^{2}}}{q^{\lambda d_{0}-d_{0}^{2}}}=\frac{1}{q} \text { for } d>d_{0}, \\
& \frac{q^{\lambda(d-1)-(d-1)^{2}}}{q^{\lambda d-d^{2}}} \leqslant \frac{q^{\lambda\left(d_{0}-1\right)-\left(d_{0}-1\right)^{2}}}{q^{\lambda d_{0}-d_{0}^{2}}}=\frac{1}{q} \text { for } d \leqslant d_{0} .
\end{aligned}
$$


This leads to claim that $\sum_{d=0}^{\infty} q^{\lambda d-d^{2}}=O\left(q^{\frac{\lambda^{2}}{4}}\right)$ since we have:

$$
\sum_{d=0}^{\infty} q^{\lambda d-d^{2}} \leqslant \sum_{d \leqslant d_{0}} q^{d-\left\lfloor d_{0}\right\rfloor} q^{\frac{\lambda^{2}}{4}}+\sum_{d>d_{0}} q^{\left\lceil d_{0}\right\rceil-d} q^{\frac{\lambda^{2}}{4}}
$$

We can use now the previous lemma together with Lemma 1 and Lemma 2 to derive the following lemma.

Lemma 5: Assuming that $\left|E_{i}\right| \geqslant r m-i$ for all $i \in\{1, \ldots, t\}$, we get:

$$
\operatorname{Pr}\left(\sum_{i=1}^{t} d\left(M_{i}\right) \geqslant u\right) \leqslant K^{t} q^{-\frac{u^{2}}{t}}
$$

where $K$ is the constant appearing in Lemma 4.

Proof: Let $D \stackrel{\text { def }}{=} \sum_{i=1}^{t} d\left(M_{i}\right)$. Using Markov's inequality:

$$
\operatorname{Pr}(D \geqslant u) \leqslant \frac{\mathbb{E}\left(q^{\lambda D}\right)}{q^{\lambda u}}
$$

for some well chosen $\lambda>0$. The exponential moment appearing at the numerator is upper-bounded with the help of the previous lemma and by using the independence of the random variables $q^{\lambda d\left(\boldsymbol{M}_{i}\right)}$ i.e.:

$$
\begin{aligned}
\mathbb{E}\left(q^{\lambda D}\right) & =\mathbb{E}\left(q^{\lambda \sum_{i=1}^{t} d\left(M_{i}\right)}\right)=\prod_{i=1}^{t} \mathbb{E}\left(q^{\lambda d\left(\boldsymbol{M}_{i}\right)}\right) \\
& \leqslant K^{t} q^{\frac{t \lambda^{2}}{4}} .
\end{aligned}
$$

Using now (21) in (20), we obtain $\operatorname{Pr}(D \geqslant \alpha t) \leqslant K^{t} \frac{q^{\frac{t \lambda^{2}}{4}}}{q^{\lambda u}}$ which implies:

$$
\operatorname{Pr}(D \geqslant \alpha t) \leqslant K^{t} q^{\frac{t \lambda^{2}}{4}-\lambda u} .
$$

We choose $\lambda=\frac{2 u}{t}$ to minimize this upper-bound leading to:

$$
\operatorname{Pr}(D \geqslant u) \leqslant K^{t} q^{-\frac{u^{2}}{t}} .
$$

The last ingredient for proving Theorem 2 is a bound on the probability that $E_{i}$ is too small to construct $F_{i}$.

Lemma 6: Let $u_{i} \stackrel{\text { def }}{=}\left(\begin{array}{c}m r \\ 2\end{array}\right)-\frac{1}{2}(2 r m-i)(i-1)$ and $F$ be the event " $\left|F_{j}\right|=r m-j$ for $j \in\{1, \ldots, i-1\}$ " then

$$
\operatorname{Pr}\left(\left|E_{i}\right|<r m-i \mid F\right) \leqslant e^{-\frac{2}{u_{i}}\left(\frac{q-1}{q} u_{i}-r m-i+1\right)^{2}} .
$$

Proof: When all the sets $F_{j}$ are of size $r m-j$ for $j$ in $\{1, \ldots, i-1\}$, it remains

$$
N-\sum_{j=1}^{i-1}(r m-j)=N-\frac{1}{2}(2 r m-i)(i-1)=u_{i}
$$

rows which can be picked up for $E_{i}$. Let $S_{t}$ be the sum of $t$ Bernoulli variables of parameter $\frac{q-1}{q}$. We obviously have

$$
\operatorname{Pr}\left(\left|E_{i}\right|<r m-i \mid F\right)=\operatorname{Pr}\left(S_{u_{i}}<r m-i\right) .
$$

It remains to use the Hoeffding inequality on the binomial tails to finish the proof.

We are ready now to prove Theorem 2: 
TABLE I

VALUES OF $r_{\max }$ AND $r_{\text {CRIT }}$ FOR A BINARY GOPPA CODE OF LENGTH $n=2^{m}$.

\begin{tabular}{c|cccccccccccccccc}
\hline$m$ & 8 & 9 & 10 & 11 & 12 & 13 & 14 & 15 & 16 & 17 & 18 & 19 & 20 & 21 & 22 & 23 \\
\hline \hline$r_{\max }$ & 5 & 8 & 8 & 11 & 16 & 20 & 26 & 34 & 47 & 62 & 85 & 114 & 157 & 213 & 290 & 400 \\
$\left\lceil r_{\text {crit }}\right\rceil$ & 5 & 6 & 8 & 11 & 14 & 19 & 25 & 34 & 46 & 62 & 84 & 114 & 156 & 214 & 293 & 402 \\
\hline
\end{tabular}

Proof of Theorem 2: Let $u=\lceil\sqrt{m r \omega(m r)})\rceil$. We observe now that if all $E_{j}$ 's are of size at least $r m-j$ for $j \in\{1, \ldots, u\}$, we can write that $D=N-\operatorname{rank}(M)$ :

$$
\begin{aligned}
D & \leqslant N-\sum_{i=1}^{r m-u} \operatorname{rank}\left(M_{i}\right) \quad \text { (by Lemma 1) } \\
& =\sum_{i=1}^{r m-1}(r m-i)-\sum_{i=1}^{r m-u} \operatorname{rank}\left(M_{i}\right) \\
& =\sum_{i=1}^{r m-u} d\left(M_{i}\right)+\sum_{i=r m-u+1}^{r m-1}(r m-i) \\
& =\sum_{i=1}^{r m-u} d\left(M_{i}\right)+\frac{u(u-1)}{2} \\
& <\sum_{i=1}^{r m-u} d\left(M_{i}\right)+\frac{1}{2} m r \omega(m r) .
\end{aligned}
$$

From this we deduce that:

$$
\operatorname{Pr}\left(D_{\text {random }} \geqslant m r \omega(m r)\right) \leqslant \operatorname{Pr}(A \cup B) \leqslant \operatorname{Pr}(A)+\operatorname{Pr}(B)
$$

where $A$ is the event " $\sum_{i=1}^{r m-u} d\left(M_{i}\right) \geqslant \frac{1}{2} m r \omega(m r)$ " and $B$ is the event "for at least one $E_{j}$ with $j \in\{1, \ldots, r m-u\}$ we have $\left|E_{j}\right|<r m-j$ ". We use now Lemma 5 to prove that $\operatorname{Pr}(A)=o(1)$ as $r m$ goes to infinity. We finish the proof by noticing that the probability of the complementary set of $B$ satisfies

$$
\begin{aligned}
\operatorname{Pr}(\bar{B}) & =\operatorname{Pr}\left(\bigcap_{i=1}^{r m-u}\left|E_{i}\right| \geqslant r m-i\right) \\
& =\prod_{i=1}^{r m-u} \operatorname{Pr}\left(\left|E_{i}\right| \geqslant r m-i \mid F\right) \\
& =1-o(1) \quad \text { (by Lemma 6). }
\end{aligned}
$$

\section{CONCLUSION AND CRYPTOGRAPHIC IMPLICATIONS}

The existence of a distinguisher for the specific case of binary Goppa codes is not valid for any value of $r$ and $m$ but tends to be true for codes that have a rate $\frac{n-m r}{n}$ very close to one. We will elaborate on this point below. This kind of codes are mainly encountered with the signature scheme [2]. If we assume that the length $n$ is equal to $2^{m}$ and we denote by $r_{\max }$ the smallest integer $r$ such that $N-T_{\text {Goppa }} \geqslant 2^{m}-m r$ then any binary Goppa code of degree $r<r_{\text {max }}$ can be distinguished (Table I). For example, the binary Goppa code obtained with $m=13$ and $r=19$ corresponding to a 90-bit security McEliece public key is distinguishable. More interestingly, all the keys proposed in [18] for the CFS signature scheme can be distinguished.

\section{A. Asymptotic Behaviour}

When the length $n$ of the code goes to infinity an asymptotic formula can be derived for the smallest rate $R_{\text {crit }}$ allowing distinguish a random code from an alternant code or a Goppa code. We derive such a formula when we assume for simplicity that the cardinality $q$ of the base field is fixed and $n$ is chosen as $n=q^{m}$ (in practice $n$ is chosen either in this way or at least of the same order as $q^{m}$ ). We also assume that the dimension $k$ of the code satisfies $k=n-r m$. Finally, we also make the assumption that the dimensions $D_{\text {alternant }}$ and $D_{\text {Goppa }}$ are given by their theoretical values $T_{\text {alternant }}$ and $T_{\text {Goppa }}$ respectively and that the dimension of $D_{\text {random }}$ is given by $T_{\text {random }} \stackrel{\text { def }}{=} \max \left(0,\left(\begin{array}{c}m r \\ 2\end{array}\right)-k\right)$. This critical rate $r_{\text {crit }}$ corresponds to the smallest 
value of $r$ for which $T_{\text {random }}$ becomes bigger than $T_{\text {alternant }}$ (asymptotically there will be no difference between Goppa codes or alternant codes). It holds that:

$$
r_{\text {crit }} \stackrel{\text { def }}{=} \min \left\{r>0 \mid T_{\text {random }} \geqslant T_{\text {alternant }}\right\}
$$

We let $R_{\text {crit }} \stackrel{\text { def }}{=} \frac{n-r_{\text {crit }} m}{n}=1-\frac{r_{\text {crit }} m}{n}$. Our claim (whose proof is postponed in the appendix) is that

Theorem 3: Let us assume that $n=q^{m}$. When $q$ is fixed and $m$ tends to infinity then $r_{\text {crit }}=\sqrt{\frac{2 q^{m} \log q}{m}}(1+o(1))$ and

$$
R_{\mathrm{crit}}=1-\sqrt{\frac{2 m \log q}{q^{m} \log m}}(1+o(1))
$$

where all logarithms are taken to base 2.

In Table I, we have computed the value of $\left[\sqrt{\frac{2 q^{m} \log q}{m \log m}}\right]$ for several $m$ ( $q$ is equal to 2 ). This shows that our approximation is rather close to $r_{\max }$ computed in practice even for small values of $m$.

\section{B. Concluding Remarks}

We emphasize that the existence of such a distinguisher does not undermine the security of McEliece [3] or CFS [2]. It only shows that the GD assumption should be used with great care. It has also been observed in [41] that the value of $D$ can be equivalently determined by considering the dimension of the square code of the dual of the public code. The square code construction relies on the component-wise product of vectors. For any vectors $\boldsymbol{a}=\left(\boldsymbol{a}_{1}, \ldots, \boldsymbol{a}_{n}\right)$ and $\boldsymbol{b}=\left(\boldsymbol{b}_{1}, \ldots, \boldsymbol{b}_{n}\right)$ we denote it by $\boldsymbol{a} \star \boldsymbol{b} \stackrel{\text { def }}{=}\left(\boldsymbol{a}_{1} \boldsymbol{b}_{1}, \ldots, \boldsymbol{a}_{n} \boldsymbol{b}_{n}\right)$. For a code $\mathscr{A}$, we denote by $\mathscr{A}^{2}$ its square code which is the linear space spanned by $\boldsymbol{a}_{i} \star \boldsymbol{a}_{j}$ where $\boldsymbol{a}_{i}$ and $\boldsymbol{a}_{j}$ describe a basis of $\mathscr{A}$. If we denote by $\mathscr{D}$ the dual of the public code then it turns out [41] that $\operatorname{dim}\left(\mathscr{D}^{2}\right)=\left(\begin{array}{c}\operatorname{dim}(\mathscr{D})+1 \\ 2\end{array}\right)-D$. It should be added that this notion has been used recently to successfully attack several cryptographic schemes relying on (modified) generalized Reed-Solomon codes [42]. More generally, a natural open question is to investigate the hardness of GD for others codes having a polynomial-time decoding algorithm (for instance, LDPC, Reed-Muller,... ).

Lastly, the recent work [30] shows that the natural reduction of GD to a hidden subgroup problem yields negligible information. As a consequence, it rules out the direct analogue of a quantum attack using the so-called Quantum Fourier Sampling (QFS) which breaks number theoretic problems [31]. More exactly, [30] shows that QFS has a negligible advantage against GD when the rate is $\geqslant R_{\mathrm{QFS}}$ where $R_{\mathrm{QFS}} \stackrel{\text { def }}{=} 1-\frac{\log _{q}(n)^{3 / 2}}{\sqrt{5 n}}=1-\frac{m^{3 / 2}}{\sqrt{5} \cdot q^{m / 2}}$. Whilst our result is somewhat contradictory with [30], it is interesting to observe that $R_{\text {crit }}$ and the critical rate $R_{\mathrm{QFS}}$ share some similarities.

\section{REFERENCES}

[1] J. Faugère, V. Gauthier-Umana, A. Otmani, L. Perret, and J. Tillich, "Distinguisher for high rate McEliece cryptosystems," in Proceedings of the 2011 IEEE Information Theory Workshop (ITW 2011), Paraty, Brazil, Oct. 16-20 2011.

[2] N. T. Courtois, M. Finiasz, and N. Sendrier, "How to achieve a McEliece-based digital signature scheme," in ASIACRYPT, vol. 2248, 2001, pp. 157-174.

[3] R. J. McEliece, A Public-Key System Based on Algebraic Coding Theory. Jet Propulsion Lab, 1978, pp. 114-116, dSN Progress Report 44.

[4] P. J. Lee and E. F. Brickell, "An observation on the security of McEliece's public-key cryptosystem," in Advances in Cryptology - EUROCRYPT'88, ser. Lecture Notes in Computer Science, vol. 330/1988. Springer, 1988, pp. 275-280.

[5] J. S. Leon, "A probabilistic algorithm for computing minimum weights of large error-correcting codes," IEEE Transactions on Information Theory, vol. 34, no. 5, pp. 1354-1359, 1988.

[6] J. Stern, "A method for finding codewords of small weight," in Coding Theory and Applications, ser. Lecture Notes in Computer Science, G. D. Cohen and J. Wolfmann, Eds., vol. 388. Springer, 1988, pp. 106-113.

[7] A. Canteaut and F. Chabaud, "A new algorithm for finding minimum-weight words in a linear code: Application to McEliece's cryptosystem and to narrow-sense BCH codes of length 511," IEEE Transactions on Information Theory, vol. 44, no. 1, pp. 367-378, 1998.

[8] D. J. Bernstein, T. Lange, and C. Peters, "Attacking and defending the McEliece cryptosystem," in PQCrypto, ser. LNCS, vol. 5299, 2008 , pp. $31-46$.

[9] _ _ "Smaller decoding exponents: Ball-collision decoding," in CRYPTO, ser. Lecture Notes in Computer Science, P. Rogaway, Ed., vol. 6841. Springer, 2011, pp. 743-760.

[10] A. May, A. Meurer, and E. Thomae, "Decoding random linear codes in $\tilde{O}\left(2^{0.054 n}\right)$," in ASIACRYPT, ser. Lecture Notes in Computer Science, D. H. Lee and X. Wang, Eds., vol. 7073. Springer, 2011, pp. 107-124.

[11] A. Becker, A. Joux, A. May, and A. Meurer, "Decoding random binary linear codes in $2^{n / 20}$ : How $1+1=0$ improves information set decoding," in EUROCRYPT, ser. Lecture Notes in Computer Science, D. Pointcheval and T. Johansson, Eds., vol. $7237 . \quad$ Springer, 2012, pp. 520-536.

[12] E. Berlekamp, R. McEliece, and H. van Tilborg, "On the inherent intractability of certain coding problems," IEEE Transactions on Information Theory, vol. 24, no. 3, pp. 384-386, May 1978.

[13] J. Gibson, "Equivalent Goppa codes and trapdoors to McElieces public key cryptosystem," in Advances in Cryptology EUROCRYPT 91, ser. Lecture Notes in Computer Science, D. Davies, Ed. Springer Berlin / Heidelberg, 1991, vol. 547, pp. 517-521.

[14] P. Loidreau and N. Sendrier, "Weak keys in the McEliece public-key cryptosystem," IEEE Transactions on Information Theory, vol. 47, no. 3, pp. 1207-1211, 2001.

[15] R. Nojima, H. Imai, K. Kobara, and K. Morozov, "Semantic security for the McEliece cryptosystem without random oracles," Des. Codes Cryptography, vol. 49, no. 1-3, pp. 289-305, 2008.

[16] R. Dowsley, J. Müller-Quade, and A. C. A. Nascimento, "A CCA2 secure public key encryption scheme based on the McEliece assumptions in the standard model," in CT-RSA, 2009, pp. 240-251.

[17] L. Dallot, "Towards a concrete security proof of Courtois, Finiasz and Sendrier signature scheme," in WEWoRC, 2007, pp. 65-77. 
[18] M. Finiasz and N. Sendrier, "Security bounds for the design of code-based cryptosystems," in Asiacrypt 2009, ser. LNCS, M. Matsui, Ed., vol. 5912. Springer, 2009, pp. 88-105.

[19] J.-C. Faugère, A. Otmani, L. Perret, and J.-P. Tillich, "Algebraic cryptanalysis of McEliece variants with compact keys," in EUROCRYPT, ser. Lecture Notes in Computer Science, H. Gilbert, Ed., vol. 6110. Springer, 2010, pp. 279-298.

[20] V. D. Goppa, "A new class of linear correcting codes," Probl. Peredachi Inf., vol. 6, no. 3, 1970.

[21] F. J. MacWilliams and N. J. A. Sloane, The Theory of Error-Correcting Codes, 5th ed. Amsterdam: North-Holland, 1986.

[22] N. Patterson, "The algebraic decoding of Goppa codes," IEEE Transactions on Information Theory, vol. 21, no. 2, pp. 203-207, 1975.

[23] J. van Tilburg, "On the McEliece public-key cryptosystem," in CRYPTO '88: Proceedings of the 8th Annual International Cryptology Conference on Advances in Cryptology. London, UK: Springer-Verlag, 1990, pp. 119-131.

[24] A. Canteaut and H. Chabanne, "A further improvement of the work factor in an attempt at breaking McEliece's cryptosystem," in EUROCODE 94. INRIA, 1994, pp. 169-173.

[25] A. Canteaut and F. Chabaud, "Improvements of the attacks on cryptosystems based on error-correcting codes," INRIA, Tech. Rep. 95-21, 1995.

[26] I. Dumer, "Suboptimal decoding of linear codes : partition techniques," IEEE Transactions on Information Theory, vol. 42, no. 6, pp. 1971-1986, 1996.

[27] A. Canteaut and N. Sendrier, "Cryptanalysis of the original McEliece cryptosystem," in Advances in Cryptology - ASIACRYPT'98, ser. Lecture Notes in Computer Sscience, no. 1514. Springer-Verlag, 1998, pp. 187-199.

[28] N. Sendrier, "On the use of structured codes in code based cryptography," in Coding Theory and Cryptography III, ser. Contactforum, S. Nikova, B. Preneel, and L. Storme, Eds. Koninklijke Vlaamse Academie van België voor Wetenschaeppen en Kunsten, 2009, pp. 59-68.

[29] — "Finding the permutation between equivalent linear codes: The support splitting algorithm," IEEE Transactions on Information Theory, vol. 46, no. 4, pp. 1193-1203, 2000.

[30] H. Dinh, C. Moore, and A. Russell, "McEliece and Niederreiter cryptosystems that resist quantum Fourier sampling attacks," in CRYPTO, ser. Lecture Notes in Computer Science, P. Rogaway, Ed., vol. 6841. Springer, 2011, pp. 761-779.

[31] P. W. Shor, "Polynomial-time algorithms for prime factorization and discrete logarithms on a quantum computer," SIAM J. Comput., vol. 26, no. 5, pp. 1484-1509, 1997.

[32] K. Kobara and H. Imai, "Semantically secure McEliece public-key cryptosystems-conversions for McEliece PKC," in Public Key Cryptography, 4th International Workshop on Practice and Theory in Public Key Cryptography, PKC 2001, Cheju Island, Korea, February 13-15, 2001, Proceedings, ser. Lecture Notes in Computer Science, K. Kim, Ed., vol. 1992. Springer, 2001, pp. 19-35.

[33] R. Nojima, H. Imai, K. Kobara, and K. Morozov, "Semantic security for the McEliece cryptosystem without random oracles," Des. Codes Cryptography, vol. 49, no. 1-3, pp. 289-305, 2008.

[34] R. Dowsley, J. Müller-Quade, and A. C. A. Nascimento, "A CCA2 secure public key encryption scheme based on the McEliece assumptions in the standard model," in CT-RSA, 2009, pp. 240-251.

[35] T. P. Berger, P. Cayrel, P. Gaborit, and A. Otmani, "Reducing key length of the McEliece cryptosystem," in Progress in Cryptology - Second International Conference on Cryptology in Africa (AFRICACRYPT 2009), ser. Lecture Notes in Computer Science, B. Preneel, Ed., vol. 5580, Gammarth, Tunisia, Jun. 21-25 2009, pp. 77-97.

[36] R. Misoczki and P. S. L. M. Barreto, "Compact McEliece keys from Goppa codes," in Selected Areas in Cryptography (SAC 2009), Calgary, Canada, Aug. 13-14 2009.

[37] J.-C. Faugère, "A new efficient algorithm for computing gröbner bases (f4)," Journal of Pure and Applied Algebra, vol. 139(1-3), pp. 61-88, 1999.

[38] — "A new efficient algorithm for computing gröbner bases without reduction to zero : F5," in ISSAC'02. ACM press, 2002, pp. 75-83.

[39] W. Bosma, J. J. Cannon, and C. Playoust, "The Magma algebra system I: The user language," J. Symb. Comput., vol. 24, no. 3/4, pp. 235-265, 1997.

[40] C. Cooper, "On the distribution of rank of a random matrix over a finite field," Random Struct. Algorithms, vol. 17, no. 3-4, pp. 197-212, 2000.

[41] I. Marquez-Corbella and R. Pellikaan, "Error-correcting pairs for a public-key cryptosystem," in CBC 2012, Code-based Cryptography Workshop 2012, 2012, paper available on http://www.win.tue.nl/ ruudp/paper/60.pdf .

[42] A. Couvreur, P. Gaborit, V. Gauthier, A. Otmani, and J. Tillich, "Distinguisher-based attacks on public-key cryptosystems using reed-solomon codes," in Proceedings of the 2013 International Workshop on Coding and Cryptography (WCC 2013), Bergen, Norway, 2013, pp. 181-193.

[43] P. Rogaway, Ed., Advances in Cryptology - CRYPTO 2011 - 31st Annual Cryptology Conference, Santa Barbara, CA, USA, August 14-18, 2011. Proceedings, ser. Lecture Notes in Computer Science, vol. 6841. Springer, 2011.

\section{APPENDIX}

\section{A. Experimental Results}

We gathered in Table II-XI some results obtained through intensive computations with the Magma system [39]. We randomly generated alternant and Goppa codes over the field $\mathbb{F}_{q}$ with $q \in\{2,4,8,16,32\}$ for values of $r$ in the range $\{3, \ldots, 50\}$ and several $m$. The Goppa codes are generated by means of an irreducible $\gamma(z)$ of degree $r$ and hence $\gamma(z)$ has no multiple roots. In particular, we can apply Theorem 1 in the binary case. We compare the dimensions of the solution space against the dimension $D_{\text {random }}$ of the system derived from a random linear code. Table II and Table III give figures for the binary case with $m=14$. We can check that $D_{\text {random }}$ is equal to 0 for $r \in\{3, \ldots, 12\}$ and $D_{\text {random }}=N-k$ as expected. We remark that $D_{\text {alternant }}$ is different from $D_{\text {random }}$ whenever $r \leqslant 15$, and $D_{\text {Goppa }}$ is different from $D_{\text {random }}$ as long as $r \leqslant 25$. Finally we observe that our formulas for $T_{\text {alternant }}$ fit as long as $k \geqslant N-D_{\text {alternant }}$ which correspond to $r \leqslant 15$. This is also the case for binary Goppa codes since we have $T_{\text {Goppa }}=D_{\text {Goppa }}$ as long as $k \geqslant N-D_{\text {Goppa }}$ i.e., $r \leqslant 25$. We also give in Table $\mathrm{X}$ and Table XI the examples we obtained for $q=4$ and $m=6$ to check that the arguments also apply. We also compare binary Goppa codes and random linear codes for $m=15$ in Table IV-VI and $m=16$ in Table VII-IX. We see that $D_{\text {random }}$ and $D_{\text {Goppa }}$ are different for $r \leqslant 33$ when $m=15$ and for $m=16$ they are different even beyond our range of experiment $(r \leqslant 50)$. 
TABLE II

$q=2$ AND $m=14$.

\begin{tabular}{|c|c|c|c|c|c|c|c|c|c|c|c|c|c|c|}
\hline $\bar{r}$ & 3 & $\overline{4}$ & 5 & $\overline{6}$ & 7 & 8 & $\overline{9}$ & 10 & 11 & 12 & 13 & 14 & 15 & 16 \\
\hline $\bar{N}$ & 861 & 1540 & 2415 & 3486 & 4753 & 6216 & 7875 & 9730 & 11781 & 14028 & 16471 & 19110 & 21945 & 24976 \\
\hline$k$ & 16342 & 16328 & 16314 & 16300 & 16286 & 16272 & 16258 & 16244 & 16230 & 16216 & 16202 & 16188 & 16174 & 16160 \\
\hline$D_{\text {random }}$ & 0 & 0 & 0 & 0 & 0 & 0 & 0 & 0 & 0 & 0 & 269 & 2922 & 5771 & 8816 \\
\hline$D_{\text {alternant }}$ & 42 & 126 & 308 & 560 & 882 & 1274 & 1848 & 2520 & 3290 & 4158 & 5124 & 6188 & 7350 & 8816 \\
\hline$T_{\text {alternant }}$ & 42 & 126 & 308 & 560 & 882 & 1274 & 1848 & 2520 & 3290 & 4158 & 5124 & 6188 & 7350 & 8610 \\
\hline$D_{\text {Goppa }}$ & 252 & 532 & 980 & 1554 & 2254 & 3080 & 4158 & 5390 & 6776 & 8316 & 10010 & 11858 & 13860 & 16016 \\
\hline$T_{\text {Goppa }}$ & 252 & 532 & 980 & 1554 & 2254 & 3080 & 4158 & 5390 & 6776 & 8316 & 10010 & 11858 & 13860 & 16016 \\
\hline
\end{tabular}

TABLE III

$q=2$ AND $m=14$.

\begin{tabular}{||c|c|c|c|c|c|c|c|c|c|c|c|c|c|c||}
\hline \hline$r$ & 17 & 18 & 19 & 20 & 21 & 22 & 23 & 24 & 25 & 26 & 27 & 28 & 29 & 30 \\
\hline \hline$N$ & 28203 & 31626 & 35245 & 39060 & 43071 & 47278 & 51681 & 56280 & 61075 & 66066 & 71253 & 76636 & 82215 & 87990 \\
\hline$k$ & 16146 & 16132 & 16118 & 16104 & 16090 & 16076 & 16062 & 16048 & 16034 & 16020 & 16006 & 15992 & 15978 & 15964 \\
\hline$D_{\text {random }}$ & 12057 & 15494 & 19127 & 22956 & 26981 & 31202 & 35619 & 40232 & 45041 & 50046 & 55247 & 60644 & 66237 & 72026 \\
\hline$D_{\text {alternant }}$ & 12057 & 15494 & 19127 & 22956 & 26981 & 31202 & 35619 & 40232 & 45041 & 50046 & 55247 & 60644 & 66237 & 72026 \\
\hline$T_{\text {alternant }}$ & 10192 & 11900 & 13734 & 15694 & 17780 & 19992 & 22330 & 24794 & 27384 & 30100 & 32942 & 35910 & 39004 & 42224 \\
\hline$D_{\text {Goppa }}$ & 18564 & 21294 & 24206 & 27300 & 30576 & 34034 & 37674 & 41496 & 45500 & 50046 & 55247 & 60644 & 66237 & 72026 \\
\hline$T_{\text {Goppa }}$ & 18564 & 21294 & 24206 & 27300 & 30576 & 34034 & 37674 & 41496 & 45500 & 49686 & 54054 & 58604 & 63336 & 68250 \\
\hline \hline
\end{tabular}

TABLE IV

$q=2$ AND $m=15$.

\begin{tabular}{||c|c|c|c|c|c|c|c|c|c|c|c|c|c|c||}
\hline \hline$r$ & 3 & 4 & 5 & 6 & 7 & 8 & 9 & 10 & 11 & 12 & 13 & 14 & 15 & 16 \\
\hline \hline$N$ & 990 & 1770 & 2775 & 4005 & 5460 & 7140 & 9045 & 11175 & 13530 & 16110 & 18915 & 21945 & 25200 & 28680 \\
\hline$k$ & 32723 & 32708 & 32693 & 32678 & 32663 & 32648 & 32633 & 32618 & 32603 & 32588 & 32573 & 32558 & 32543 & 32528 \\
\hline$D_{\text {random }}$ & 0 & 0 & 0 & 0 & 0 & 0 & 0 & 0 & 0 & 0 & 0 & 0 & 0 & 0 \\
\hline$D_{\text {Goppa }}$ & 270 & 570 & 1050 & 1665 & 2415 & 3300 & 4455 & 5775 & 7260 & 8910 & 10725 & 12705 & 14850 & 17160 \\
\hline$T_{\text {Goppa }}$ & 270 & 570 & 1050 & 1665 & 2415 & 3300 & 4455 & 5775 & 7260 & 8910 & 10725 & 12705 & 14850 & 17160 \\
\hline \hline
\end{tabular}

TABLE V

$q=2$ AND $m=15$

\begin{tabular}{||c|c|c|c|c|c|c|c|c|c|c|c|c|c|c||}
\hline \hline$r$ & 17 & 18 & 19 & 20 & 21 & 22 & 23 & 24 & 25 & 26 & 27 & 28 & 29 & 30 \\
\hline \hline$N$ & 32385 & 36315 & 40470 & 44850 & 49455 & 54285 & 59340 & 64620 & 70125 & 75855 & 81810 & 87990 & 94395 & 101025 \\
\hline$k$ & 32513 & 32498 & 32483 & 32468 & 32453 & 32438 & 32423 & 32408 & 32393 & 32378 & 32363 & 32348 & 32333 & 32318 \\
\hline$D_{\text {random }}$ & 0 & 3817 & 7987 & 12382 & 17002 & 21847 & 26917 & 32212 & 37732 & 43477 & 49447 & 55642 & 62062 & 68707 \\
\hline$D_{\text {Goppa }}$ & 19890 & 22815 & 25935 & 29250 & 32760 & 36465 & 40365 & 44460 & 48750 & 53235 & 57915 & 62790 & 67860 & 73125 \\
\hline$T_{\text {Goppa }}$ & 19890 & 22815 & 25935 & 29250 & 32760 & 36465 & 40365 & 44460 & 48750 & 53235 & 57915 & 62790 & 67860 & 73125 \\
\hline \hline
\end{tabular}

TABLE VI

$q=2$ AND $m=15$.

\begin{tabular}{||c|c|c|c|c|c|c|c|c|c|c|c|c|c|c||}
\hline \hline$r$ & 31 & 32 & 33 & 34 & 35 & 36 & 37 & 38 & 39 & 40 & 41 & 42 & 43 & 44 \\
\hline \hline$N$ & 107880 & 114960 & 122265 & 129795 & 137550 & 145530 & 153735 & 162165 & 170820 & 179700 & 188805 & 198135 & 207690 & 217470 \\
\hline$k$ & 32303 & 32288 & 32273 & 32258 & 32243 & 32228 & 32213 & 32198 & 32183 & 32168 & 32153 & 32138 & 32123 & 32108 \\
\hline$D_{\text {random }}$ & 75577 & 82672 & 89992 & 97537 & 105307 & 113302 & 121522 & 129967 & 138637 & 147532 & 156652 & 165997 & 175567 & 185362 \\
\hline$D_{\text {Goppa }}$ & 78585 & 84240 & 90585 & 97537 & 105307 & 113302 & 121522 & 129967 & 138637 & 147532 & 156652 & 165997 & 175567 & 185362 \\
\hline$T_{\text {Goppa }}$ & 78585 & 84240 & 90585 & 97155 & 103950 & 110970 & 118215 & 125685 & 133380 & 141300 & 149445 & 157815 & 166410 & 175230 \\
\hline \hline
\end{tabular}

\section{B. Proof of Theorem 3}

To prove Theorem 3 we will first use the following observation 
TABLE VII

$q=2$ AND $m=16$.

\begin{tabular}{|c|c|c|c|c|c|c|c|c|c|c|c|c|c|c|}
\hline$r$ & 3 & 4 & 5 & 6 & 7 & 8 & 9 & 10 & 11 & 12 & 13 & 14 & 15 & 16 \\
\hline $\bar{N}$ & 1128 & 2016 & 3160 & 4560 & 6216 & 8128 & 10296 & 12720 & 15400 & 18336 & 21528 & 24976 & 28680 & 32640 \\
\hline$k$ & 65488 & 65472 & 65456 & 65440 & 65424 & 65408 & 65392 & 65376 & 65360 & 65344 & 65328 & 65312 & 65296 & 65280 \\
\hline$D_{\text {random }}$ & 0 & 0 & 0 & 0 & 0 & 0 & 0 & 0 & 0 & 0 & 0 & 0 & 0 & 0 \\
\hline$D_{\text {Goppa }}$ & 288 & 608 & 1120 & 1776 & 2576 & 3520 & 4752 & 6160 & 7744 & 9504 & 11440 & 13552 & 15840 & 18304 \\
\hline$T_{\text {Goppa }}$ & 288 & 608 & 1120 & 1776 & 2576 & 3520 & 4752 & 6160 & 7744 & 9504 & 11440 & 13552 & 15840 & 18304 \\
\hline
\end{tabular}

TABLE VIII

$q=2$ AND $m=16$.

\begin{tabular}{||c|c|c|c|c|c|c|c|c|c|c|c|c|c|c||}
\hline \hline$r$ & 17 & 18 & 19 & 20 & 21 & 22 & 23 & 24 & 25 & 26 & 27 & 28 & 29 & 30 \\
\hline \hline$N$ & 36856 & 41328 & 46056 & 51040 & 56280 & 61776 & 67528 & 73536 & 79800 & 86320 & 93096 & 100128 & 107416 & 114960 \\
\hline$k$ & 65264 & 65248 & 65232 & 65216 & 65200 & 65184 & 65168 & 65152 & 65136 & 65120 & 65104 & 65088 & 65072 & 65056 \\
\hline$D_{\text {random }}$ & 0 & 0 & 0 & 0 & 0 & 0 & 2360 & 8384 & 14664 & 21200 & 27992 & 35040 & 42344 & 49904 \\
\hline$D_{\text {Goppa }}$ & 21216 & 24336 & 27664 & 31200 & 34944 & 38896 & 43056 & 47424 & 52000 & 56784 & 61776 & 66976 & 72384 & 78000 \\
\hline$T_{\text {Goppa }}$ & 21216 & 24336 & 27664 & 31200 & 34944 & 38896 & 43056 & 47424 & 52000 & 56784 & 61776 & 66976 & 72384 & 78000 \\
\hline \hline
\end{tabular}

TABLE IX

$q=2$ AND $m=16$.

\begin{tabular}{|c|c|c|c|c|c|c|c|c|c|c|c|c|c|}
\hline $\bar{r}$ & 31 & 32 & 33 & 34 & 35 & 36 & 37 & 38 & 39 & 40 & 41 & 42 & 43 \\
\hline$\overline{N N}$ & 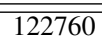 & 130816 & 139128 & 147696 & $\overline{0156520}$ & 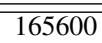 & 1744936 & 184528 & 194376 & 204480 & 214840 & 225456 & 236328 \\
\hline$k$ & 65040 & 65024 & 65008 & 64992 & 64976 & 64960 & 64944 & 64928 & 64912 & 64896 & 64880 & 64864 & 64848 \\
\hline$D_{\text {random }}$ & 57720 & 65792 & 74120 & 82704 & 91544 & 100640 & 109992 & 119600 & 129464 & 139584 & 149960 & 160592 & 171480 \\
\hline$T_{\text {Goppa }}$ & 83824 & 89856 & 96624 & 103632 & 110880 & 118368 & 126096 & 134064 & 142272 & 150720 & 159408 & 168336 & 177504 \\
\hline
\end{tabular}

TABLE X

$q=4$ AND $m=6$.

\begin{tabular}{||c|c|c|c|c|c|c|c|c|c|c|c|c|c|c||}
\hline \hline$r$ & 3 & 4 & 5 & 6 & 7 & 8 & 9 & 10 & 11 & 12 & 13 & 14 & 15 & 16 \\
\hline \hline$N$ & 153 & 276 & 435 & 630 & 861 & 1128 & 1431 & 1770 & 2145 & 2556 & 3003 & 3486 & 4005 & 4560 \\
\hline$k$ & 4078 & 4072 & 4066 & 4060 & 4054 & 4048 & 4042 & 4036 & 4030 & 4024 & 4018 & 4012 & 4006 & 4000 \\
\hline$D_{\text {random }}$ & 0 & 0 & 0 & 0 & 0 & 0 & 0 & 0 & 0 & 0 & 0 & 0 & 0 & 560 \\
\hline$D_{\text {alternant }}$ & 6 & 18 & 60 & 120 & 198 & 294 & 408 & 540 & 690 & 858 & 1044 & 1248 & 1470 & 1710 \\
\hline$T_{\text {alternant }}$ & 6 & 18 & 60 & 120 & 198 & 294 & 408 & 540 & 690 & 858 & 1044 & 1248 & 1470 & 1710 \\
\hline$D_{\text {Goppa }}$ & 18 & 60 & 120 & 198 & 294 & 408 & 540 & 750 & 990 & 1260 & 1560 & 1890 & 2250 & 2640 \\
\hline$T_{\text {Goppa }}$ & 18 & 60 & 120 & 198 & 294 & 408 & 540 & 750 & 990 & 1260 & 1560 & 1890 & 2250 & 2640 \\
\hline \hline
\end{tabular}

TABLE XI

$q=4$ AND $m=6$.

\begin{tabular}{||c|c|c|c|c|c|c|c|c|c|c|c|c|c|c||}
\hline \hline$r$ & 17 & 18 & 19 & 20 & 21 & 22 & 23 & 24 & 25 & 26 & 27 & 28 & 29 & 30 \\
\hline \hline$N$ & 5151 & 5778 & 6441 & 7140 & 7875 & 8646 & 9453 & 10296 & 11175 & 12090 & 13041 & 14028 & 15051 & 16110 \\
\hline$k$ & 3994 & 3988 & 3982 & 3976 & 3970 & 3964 & 3958 & 3952 & 3946 & 3940 & 3934 & 3928 & 3922 & 3916 \\
\hline$D_{\text {random }}$ & 1157 & 1790 & 2459 & 3164 & 3905 & 4682 & 5495 & 6344 & 7229 & 8150 & 9107 & 10100 & 11129 & 12194 \\
\hline$D_{\text {alternant }}$ & 2064 & 2448 & 2862 & 3306 & 3905 & 4682 & 5495 & 6344 & 7229 & 8150 & 9107 & 10100 & 11129 & 12194 \\
\hline$T_{\text {alternant }}$ & 2064 & 2448 & 2862 & 3306 & 3780 & 4284 & 4818 & 5382 & 5976 & 6600 & 7254 & 7938 & 8652 & 9396 \\
\hline$D_{\text {Goppa }}$ & 3060 & 3510 & 3990 & 4500 & 5040 & 5610 & 6210 & 6840 & 7500 & 8190 & 9107 & 10100 & 11129 & 12194 \\
\hline$T_{\text {Goppa }}$ & 3060 & 3510 & 3990 & 4500 & 5040 & 5610 & 6210 & 6840 & 7500 & 8190 & 8910 & 9660 & 10440 & 11250 \\
\hline \hline
\end{tabular}

Lemma 7: Let $T_{\text {alternant }}$ be as defined in (4). Let also $T_{\text {Goppa }}$ be as defined in (6). There exists constants $K_{1}$ and $K_{2}$ (resp. $K_{1}^{\prime}$ and $K_{1}^{\prime}$ and $K_{2}^{\prime}$ ) such that:

$$
\begin{gathered}
m r^{2}\left(\log _{q}(r)+K_{1}\right) \leqslant T_{\text {alternant }} \leqslant m r^{2}\left(\log _{q}(r)+K_{2}\right), \\
m r^{2}\left(\log _{q}(r)+K_{1}^{\prime}\right) \leqslant T_{\text {Goppa }} \leqslant m r^{2}\left(\log _{q}(r)+K_{2}^{\prime}\right) .
\end{gathered}
$$


Proof: We recall that:

$$
T_{\text {alternant }} \stackrel{\text { def }}{=} \frac{1}{2} m(r-1)\left((2 e+1) r-2 \frac{q^{e+1}-1}{q-1}\right)
$$

where $e \stackrel{\text { def }}{=}\left\lfloor\log _{q}(r-1)\right\rfloor$. There exists some absolute constants $K_{3}$ and $K_{4}$ such that for all integers $r \geqslant 2$ :

$$
\left\{\begin{array}{l}
2 r \log _{q}(r)+K_{3} r \leqslant(2 e+1) r-2 \frac{q^{e+1}-1}{q-1} \\
2 r \log _{q}(r)+K_{4} r \geqslant(2 e+1) r-2 \frac{q^{e+1}-1}{q-1}
\end{array}\right.
$$

The upper bound is clear since:

$$
(2 e+1) r-2 \frac{q^{e+1}-1}{q-1} \leqslant 2 r \log _{q}(r)+2 r .
$$

For the lower bound, we remark that:

$$
e \geqslant \log _{q}(r-1)-1=\log _{q}(r)+\log _{q}(1-1 / r)-1 .
$$

In addition:

$$
\frac{q^{e+1}-1}{q-1} \leqslant q \cdot q^{e} \leqslant r q .
$$

As a consequence $\left((2 e+1) r-2 \frac{q^{e+1}-1}{q-1}\right) \geqslant 2 r \log _{q}(r)+r\left(2 \log _{q}(1-1 / r)-1-2 q\right)$. Remark that $\log _{q}(1-1 / r)$ can be bounded from above by some (negative) constant. So, it holds that:

$$
\left((2 e+1) r-2 \frac{q^{e+1}-1}{q-1}\right) \geqslant 2 r \log _{q}(r)+K_{3} r,
$$

for some constant $K_{3}$. Observe now that $\frac{1}{2} m(r-1)\left(2 \log _{q}(r)+K_{3} r\right)=\frac{1}{2}(m r-m)\left(2 \log _{q}(r)+K_{3} r\right)$ is equal to:

$$
\frac{1}{2}\left(2 m r \log _{q}(r)+K_{3} m r^{2}-2 m \log _{q}(r)-K_{3} m r\right) .
$$

The lower bound on $T_{\text {alternant }}$ follows immediately from this. The expression can be lower bounded (resp. upper bounded) by a term of the form $K_{1} m r^{2}$ (resp. $K_{2} m r^{2}$ ) for some constant $K_{1}$ (resp. $K_{2}$ ). This holds for all positive integers $r$. Finally, we recall that when $r<q-1$ :

$$
T_{\text {Goppa }}=T_{\text {alternant }}=\frac{1}{2} m(r-1)(r-2)
$$

and when $r \geqslant q-1$ :

$$
T_{\text {Goppa }}=\frac{1}{2} m r\left((2 e+1) r-2(q-1) q^{e-1}-1\right) .
$$

with $e$ being the unique integer such that:

$$
(q-1)^{2} q^{e-2}<r \leqslant(q-1)^{2} q^{e-1} .
$$

The bound (23) on can be proved in the same way.

From this lemma, we deduce that:

Lemma 8: There exist two constants $C_{1}$ and $C_{2}$ such that for every $r$ satisfying $\left(\begin{array}{c}m r \\ 2\end{array}\right) \geqslant n-m r$ we have:

$$
\begin{aligned}
& m r^{2}\left(m / 2-\log _{q}(r)+C_{1}\right)-q^{m} \leqslant T_{\text {random }}-T_{\text {alternant }}, \\
& m r^{2}\left(m / 2-\log _{q}(r)+C_{2}\right)-q^{m} \geqslant T_{\text {random }}-T_{\text {alternant }} .
\end{aligned}
$$

We also have the same inequalities when we replace $T_{\text {random }}-T_{\text {alternant }}$ with $T_{\text {random }}-T_{\text {Goppa }}$.

Proof: For all positive integer values of $r$ such that $\left(\begin{array}{c}m r \\ 2\end{array}\right) \geqslant n-m r$, we have:

$$
\begin{aligned}
T_{\text {random }} & =N-k=\left(\begin{array}{c}
m r \\
2
\end{array}\right)-q^{m}+m r \\
& =m^{2} r^{2} / 2-q^{m}+m r / 2 .
\end{aligned}
$$

We can then conclude using Lemma 7.

From this lemma, we derive the following estimate for $r_{\text {crit }}$ :

Lemma 9: When $m$ goes to infinity we have

$$
r_{\text {crit }}=\sqrt{\frac{2 q^{m} \log q}{m \log m}}(1+o(1)) .
$$


Proof: From Lemma 8, we know that:

$$
T_{\text {random }}-T_{\text {alternant }}=m r^{2}\left(m / 2-\log _{q}(r)\right)-q^{m}+O\left(m r^{2}\right) .
$$

Let $r_{0} \stackrel{\text { def }}{=} \sqrt{\frac{2 q^{m} \log q}{m \log m}}$. It holds that:

$$
\begin{aligned}
2 \log _{q}\left(r_{0}\right) & =\log _{q}\left(2 q^{m} \log q\right)-\log _{q}(m \log m), \\
& =m+\log _{q}(2 \log q)-\log _{q}(m)-\log _{q}(\log m) .
\end{aligned}
$$

Thus by writing that $m r_{0}^{2}\left(m / 2-\log _{q} r_{0}\right)-q^{m}$ is equal to:

$$
\begin{aligned}
\frac{2 q^{m} \log q}{\log m} & (m / 2-m / 2 \\
& \left.-\frac{\log (2 \log q)}{2 \log q}+\frac{\log m}{2 \log q}+\frac{\log \log m}{2 \log q}\right)-q^{m}
\end{aligned}
$$

which in turn equals $\frac{q^{m}}{\log m}(\log \log m-\log (2 \log q))$. We also observe that $m r_{0}^{2}=\frac{2 q^{m} \log q}{\log m}$ is negligible compared to $\frac{q^{m}}{\log m}(\log \log m-\log ($ when $m$ goes to infinity. This can be used to show that $T_{\text {random }}-T_{\text {alternant }}$ is positive for $r=\left\lceil r_{0}\right\rceil$ when $m$ is large enough. Therefore for $m$ large enough, we have $r_{\text {crit }} \leqslant\left\lceil r_{0}\right\rceil$. On the other hand, let $\alpha$ be any positive constant $<1$. We set:

$$
r_{\alpha} \stackrel{\text { def }}{=} \sqrt{\frac{2 \alpha q^{m} \log q}{m \log m}} .
$$

Notice that the function $f(x)=m x^{2}\left(m / 2-\log _{q} x\right)-q^{m}$ can be shown to be increasing in the range $\left(0, r_{\alpha}\right)$. Therefore for every $r \leqslant r_{\alpha}$, we have $m r^{2}\left(m / 2-\log _{q} r\right)-q^{m}$ is less than or equal to:

$$
\begin{aligned}
-q^{m}+\frac{2 \alpha q^{m} \log q}{\log m} & \left(m / 2-m / 2+\frac{\log m}{2 \log q}\right. \\
+ & \left.\frac{\log \log m}{2 \log q}-\frac{\log (2 \alpha \log q)}{2 \log q}\right)
\end{aligned}
$$

This last quantity is upper-bounded by:

which in turn is less than or equal to:

$$
(\alpha-1) q^{m}+\frac{2 \alpha q^{m} \log q}{\log m}\left(\frac{\log \log m}{2 \log q}-\frac{\log (2 \alpha \log q)}{2 \log q}\right)
$$

$$
(\alpha-1) q^{m}+q^{m} \frac{\alpha \log \log m}{\log m} .
$$

Since $m r^{2} \leqslant \frac{2 \alpha q^{m} \log q}{\log m}$, it follows that any function of the form $m r^{2}\left(m / 2-\log _{q} r\right)-q^{m}+O\left(m r^{2}\right)$ will be negative for $m$ large enough in the range $\left(0, r_{\alpha}\right)$. This implies that $r_{\text {crit }} \geqslant r_{\alpha}=\sqrt{\frac{2 \alpha q^{m} \log q}{m \log m}}$ for $m$ large enough. We deduce from this fact which holds for any $0<\alpha<1$ and from the upper bound $r_{\text {crit }} \leqslant\left\lceil r_{0}\right\rceil=\left\lceil\sqrt{\frac{2 q^{m} \log q}{m \log m}}\right\rceil$ that $r_{\text {crit }}=\sqrt{\frac{2 q^{m} \log q}{m \log m}}(1+o(1))$ when $m$ goes to infinity. Finally, the proof of Theorem 3 is now obtained by remarking:

$$
R_{\text {crit }}=\frac{q^{m}-m r_{\text {crit }}}{q^{m}}=1-\sqrt{\frac{2 m \log q}{q^{m} \log m}}(1+o(1)) .
$$

Jean-Charles Faugère Jean-Charles Faugère graduated from École Normale Supérieure, Paris, France, and received a Ph.D. degree from the University of Paris 6 in 1994. He was a CNRS Researcher until 2006; presently, he is a INRIA Senior Researcher and the head of the project-team PolSys which is common between INRIA (Paris-Rocquencourt), UPMC (LIP6) and CNRS (LIP6). His research interests are in computer algebra, polynomial systems solving, Gröbner basis, cryptography and other applications of Computer Algebra.

Valérie Gauthier-Umaña was born in Lyon, France. She received a M.Sc. degree (ALGANT program) in mathematics from the University of Bordeaux I, France and the Univertià degli study di Padova, Padova, Italy in 2008, and a Ph.D. degree in mathematics from the Technical University of Denmark, Lyngby, Denmark in 2011. In 2012 she was a postdoctoral Researcher at the University of Caen, France. She is currently a postdoctoral Researcher at the Universidad de los Andes, Bogotá, Colombia. Her research lies in cryptography and coding theory. 
Ayoub Otmani holds a Ph.D. degree in mathematics and its applications from the University of Limoges (France) since 2002. From 2004 through 2012 he was associate professor at Ensicaen (Caen, France). From 2009 to 2011, he was in secondment as a Researcher at the Institut de Recherche en Informatique et Automatique (INRIA - Rocquencourt) He is currently Professor at University of Rouen, France. His research lies in the fields of algebraic coding theory and cryptography.

Ludovic Perret holds a Ph.D. degree in computer science from the University of Marne-la-Vallée (France) since 2005. He is associate professor since 2007 at UPMC (Paris, France) in the project-team PolSys which is common between UPMC/LIP6 and INRIA-Rocquencourt. His research area is at the intersection of cryptography and symbolic computation.

Jean-Pierre Tillich (M'06) received the Engineer degree from École des Mines de Paris, Paris, France, in 1989 and the Ph.D. degree in computer science from École Nationale Suprieure des Télćommunications (ENST), Paris, in 1994. From 1997 to 2003, he was an Assistant Professor at the University Paris $\mathrm{XI}$. He is now a Researcher at the Institut de Recherche en Informatique et Automatique (INRIA), Rocquencourt, Le Chesnay, France. His research interests include classical and quantum coding theory, cryptography and graph theory. 